\title{
Multicomponent stress-strength reliability based on a right long-tailed distribution
}

\author{
Hossein Pasha-Zanoosi (D), Ahmad Pourdarvish* \\ Department of Statistics, University of Mazandaran, Babolsar, Iran
}

\begin{abstract}
This article deals with the problem of reliability in a multicomponent stress-strength (MSS) model when both stress and strength variables are from inverse Kumaraswamy distribution. The reliability of the system is estimated using classical and Bayesian approaches when the common second shape parameter is known or unknown. The maximum likelihood estimation and its asymptotic confidence interval for the reliability of the system are obtained. Furthermore, two other asymptotic confidence intervals are computed based on Logit and Arcsin transformations. The uniformly minimum variance unbiased estimator for the reliability of the MSS model is obtained when the common second shape parameter is known. The Bayes estimate is obtained exactly when the second shape parameter is known and it is approximated by using the Monte Carlo Markov Chain method when the second shape parameter is unknown. The highest probability density credible interval is established using the Gibbs sampling technique. Monte Carlo simulations are implemented to compare the different proposed methods. Finally, two real data sets are presented in support of the suggested procedures.
\end{abstract}

Mathematics Subject Classification (2020). 62N05, 62F10, 62F12, 62F15

Keywords. Inverse Kumaraswamy distribution, right long-tailed distribution, multicomponent stress-strength model

\section{Introduction}

The Kumaraswamy distribution, introduced by [28], is one of the most important lifetime distributions with finite support. However, it can not be used for most lifetime data that have infinite support in theory. The probability density function (pdf) of the Kumaraswamy distribution with two positive shape parameters $\alpha$ and $\theta$ is specified by

$$
f(z ; \alpha, \theta)=\alpha \theta z^{\theta-1}\left[1-z^{\theta}\right]^{\alpha-1}, \quad 0<z<1 .
$$

To analyze lifetime data sets in the best-case scenario, the inverse Kumaraswamy (IKu) distribution introduced by [1], is very flexible.

\footnotetext{
${ }^{*}$ Corresponding Author.

Email addresses: pashazanoosi@yahoo.com (H. Pasha-Zanoosi), a.pourdarvish@umz.ac.ir (A. Pourdarvish)

Received: 16.02.2021; Accepted: 20.01.2022
} 
The IKu distribution corresponds to the distribution of the random variable $X=1 / Z-1$ where $Z$ has a Kumaraswamy distribution with parameters $\alpha$ and $\theta$. The transformation $X=1 / Z-1$ is more appropriate than the transformation $X=1 / Z$ which makes it more flexible for modeling lifetime data. The cumulative distribution function (cdf) of IKu distribution is as follows:

$$
F_{X}(x ; \alpha, \theta)=\left[1-(1+x)^{-\theta}\right]^{\alpha}, \quad x>0
$$

and the corresponding pdf is

$$
f_{X}(x ; \alpha, \theta)=\alpha \theta(1+x)^{-(\theta+1)}\left[1-(1+x)^{-\theta}\right]^{\alpha-1}, \quad x>0,
$$

where $\alpha>0$ and $\theta>0$ are first and second shape parameters, respectively. Hereafter, we use the notation $\operatorname{IKu}(\alpha, \theta)$ for the random variable that has an IKu distribution with parameters $\alpha$ and $\theta$. The several distributional properties of the IKu distribution were discussed in [1]. The curves for pdf and hazard rate function (hrf) of the IKu distribution reveal decreasing (monotonic) and upside-down bathtub (non-monotonic) shapes (Figures 1 and 2). The estimation and prediction problems of this distribution based on general progressive censored samples were considered in [2]. A generalized version of the IKu distribution was proposed in [20], while a bivariate generalization of the IKu distribution was studied in [34].

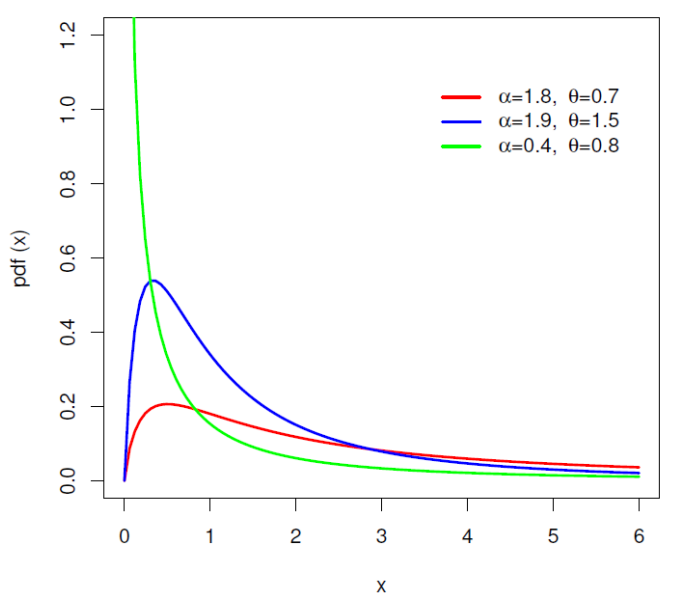

Figure 1. The pdf plots of the IKu distribution for different combinations of $(\alpha, \theta)$.

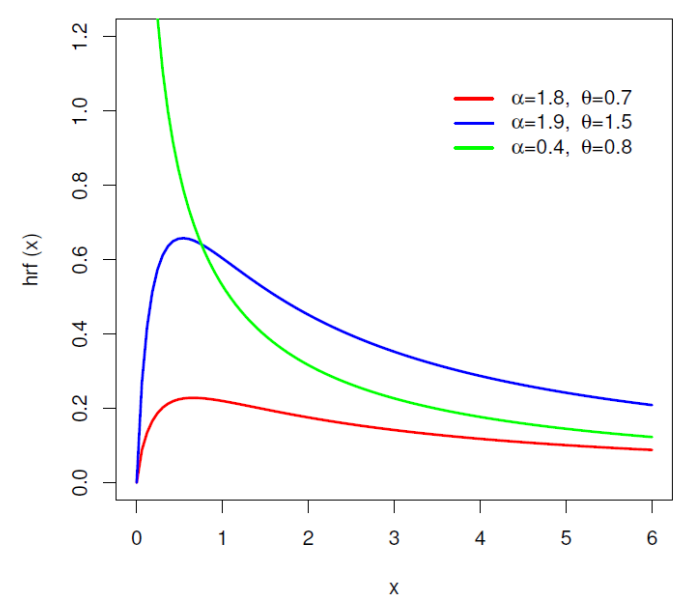

Figure 2. The hrf plots of the IKu distribution for different combinations of $(\alpha, \theta)$. 
In probability theory, long-tailed distributions are probability distributions whose tails asymptotically approach zero at a slower than one with exponential tails. They tend to have many outliers with very large observations. In the context of reliability, the extreme outlying observations in the direction of the right tail are important, however, a distribution may have a long left tail, or both tails may be long. The distribution of a random variable $X$ with cdf of $F$ is said to have a long tail if $F$ has right unbounded support and for all $\mathrm{t}>0$,

$$
\lim _{x \rightarrow \infty} \frac{1-F(x+t)}{1-F(x)}=1 .
$$

Long-tailed distributions are a subclass of heavy-tailed distributions. All long-tailed distributions are heavy-tailed, but the opposite may not be true. For detailed information about the classification of probability distributions based on their tail behavior, one could refer to $[16,42-44,48]$. The class of long-tailed distributions includes several well-known lifetimes such as Pareto, Log-normal, Burr Type XII distributions, and so on. Long-tailed distributions have many important applications in many fields including meteorology, aerospace engineering, insurance, and finance. For example, commerce and marketing plans usually find that their sales can best be described using the long-tailed distributions. Consider an internet store. It may have certain items with very high sales and a large number of items with much fewer sales. Although the sales volume for every individual item at the far end of the tail may be negligible, there are enough items that play an important role in the general profit-taking. The profit from low-sale volume items can compete with the profit gained from best sellers.

Some of the attractive features of the IKu distribution are as follows:

- The IKu distribution belongs to the class of long-tailed distributions [1]. It has a longer tail than several commonly used distributions, and this feature positively affects the capability of distribution to fit the rare events (outlying observations) occurring in the direction of the right tail. This means that the IKu distribution can be considered as an extreme value distribution. Many random variables have long-tailed distributions, including traffic patterns on the internet, city population sizes, natural resource occurrences, stock price fluctuations, company sizes, income, and so on. Therefore, such data can be modeled using the IKu distribution.

- The IKu distribution is known as the inverse version of a probability distribution. The study of inverse distributions has provided a better understanding of standard distributions and has helped increase flexibility for data fitting [30]. It is surprising and interesting to know the inverse versions of the probability distributions indicate the upside-down bathtub shapes for their hazard rates [46]. From a practical perspective, there are many cases in which the data sets show upside-down bathtub hazard rate behavior. One such instance was analyzed by [15] within the scope of head and neck cancer data, in which the hazard rate firstly increased, attended a maximum, and then decreased before it ultimately stabilized due to therapy. As another instance, Langlands et al. [29] have investigated the data of breast carcinoma and observed that the mortality increased firstly, got to a peak, and then decreased.

- The IKu distribution belongs to proportional reversed hazard models that are an important class of distribution families in reliability and survival analysis. This family of distributions is defined as $F_{X}(x)=\left[F_{0}(x)\right]^{\alpha}$, where $\alpha$ is positive and $F_{0}($.$) is the cdf of the baseline random variable.$

What distinguishes the IKu distribution from competing distributions is that, in addition to having a long tail, it is also known as the inverse version of a probability distribution, as well as a model with a proportional reversed hazard rate. The IKu distribution is a better fit than six competitive models, including two long-tailed distributions (Burr Type 
XII and modified Lomax), two inverse distributions (inverse Weibull and inverse Gamma) and two proportional reversed hazard models (exponentiated Weibull and exponentiated Rayleigh) based on two real data sets (see Section 5). These justify the motivation for an applied study based on the IKu distribution.

The estimation problem of stress-strength reliability, $R=P(Y<X)$, has been extensively investigated in the statistical literature. It has a broad application in many fields such as engineering, agriculture, medical and so on. Here, $X$ presents the strength of a system that is exposed to a stress $Y$. Thus $R$ indicates the system performance. In engineering applications, if $X$ denotes the strength of a building and $Y$ represents the resultant of the destructive forces acting on it, such as an earthquake, then $R$ can be interpreted as the safety factor of a building. In aquaculture, if $X$ is the growth value of fish in a treatment group and $Y$ is the growth value of a control group, then $R$ shows the effectiveness of treatment. This fundamental idea was firstly studied in [11]. Thereafter, the problem of estimating $R$ has been discussed by a great number of researchers. Some recent efforts pertaining to stress-strength models can be found in $[5-9,18,41]$ for the Weibull, Lindley, Dagum, truncated proportional hazard rate family, proportional hazard rate family, Topp-Leone, and generalized Pareto distributions, respectively.

In recent years, inference for the reliability of the MSS model has received much attention among researchers. This model contains $k$ identical and independent strength components and it operates when at least $s(1 \leq s \leq k)$ of the components work properly against common stress. It is commonly known as $s$-out-of- $k$ : G system. MSS models appear in many practical situations, such as communication systems, industrial operations, military technologies, and so on. For example, consider an airplane with four engines that flies when at least two engines work satisfactorily. Thus, the airplane operation is a 2-out-of-4: G system. As another example, the kidney function in the human body is a 1-out-of-2: $\mathrm{G}$ system, since a person can survive with at least one healthy kidney. Assume $X_{1}, X_{2}, \ldots, X_{k}$ are independent random variables with common cdf of $F($.$) and exposed to$ the common stress $Y$ with cdf of $G($.$) , Thus, the reliability in the MSS model is given by$

$$
\begin{aligned}
R_{s, k} & =P\left[\text { at least } s \text { of }\left(X_{1}, X_{2}, \ldots, X_{k}\right) \text { exceed } Y\right] \\
& =\sum_{i=s}^{k}\left(\begin{array}{c}
k \\
i
\end{array}\right) \int_{0}^{\infty}\left[1-F_{X}(y)\right]^{i}\left[F_{X}(y)\right]^{k-i} d G(y) .
\end{aligned}
$$

The mentioned model was firstly examined in [10]. Thereafter, many authors have shown considerable interest in the MSS model. Some recent efforts regard to the issue, can be found in $[3,4,14,21-27,31-33,37]$ for the Topp-Leone, exponentiated Pareto distribution, Kumaraswamy, unit-Gompertz, unit generalized Rayleigh, Geometric, Chen, proportional reversed hazard rate family, bivariate Kumaraswamy, Weibull, the general class of inverted Exponentiated models, inverted exponentiated Rayleigh, Burr XII, and bathtub-shaped distributions, respectively.

To our knowledge, until now, no work has been carried out to study the MSS model under the IKu distribution. The focus of this article is to establish classical and Bayesian inferences on the reliability of the MSS model when the stress and the strength both follow the IKu distribution. The rest of the content of this paper is organized as follows. In Section 2, when the common second shape parameter is unknown, the maximum likelihood estimate (MLE) of $R_{s, k}$ along with its asymptotic confidence interval (ACI) are obtained. Also, two other asymptotic confidence intervals are constructed based on Logit and Arcsin transformations. The Bayes estimation of $R_{s, k}$ is obtained by using the MCMC method under square loss (SE) function. Furthermore, the highest probability density (HPD) credible interval is provided in this section. In Section 3, when the second shape parameter is known, the MLE, uniformly minimum variance unbiased estimate (UMVUE), and ACI of $R_{s, k}$ are investigated. The Bayes estimator of $R_{s, k}$ is determined explicitly, in this 
section. Moreover, the HPD credible interval is provided. In Section 4, proposed methods are compared via Monte Carlo simulations. In Section 5, the analysis of two real data sets is provided for a demonstration of the findings. Finally, concluding remarks are considered in Section 6.

\section{Estimation of $R_{s, k}$ when $\theta$ is unknown}

\subsection{MLE of $\boldsymbol{R}_{s, k}$}

Suppose $X_{1}, X_{2}, \ldots, X_{k}$ be independent strength random variables that follow $I K u(\alpha, \theta)$ and $Y$ be stress random variable follows $\operatorname{IKu}(\beta, \theta)$. Hence, the reliability of the MSS model, using the Equations (1.1), (1.2), and (1.3) is obtained as

$$
\begin{aligned}
R_{s, k}=\sum_{i=s}^{k}\left(\begin{array}{c}
k \\
i
\end{array}\right) \beta & \int_{0}^{\infty}\left[1-\left\{1-(1+y)^{-\theta}\right\}^{\alpha}\right]^{i}\left[1-(1+y)^{-\theta}\right]^{\alpha(k-i)} \theta(1+y)^{-(\theta+1)} \\
& \times\left[1-(1+y)^{-\theta}\right]^{\beta-1} d y
\end{aligned}
$$

By using the change of variable $z=1-(1+y)^{-\theta}$, we have

$$
\begin{aligned}
R_{s, k} & =\sum_{i=s}^{k}\left(\begin{array}{c}
k \\
i
\end{array}\right) \beta \int_{0}^{1}\left(1-z^{\alpha}\right)^{i} z^{\alpha(k-i)+\beta-1} d z \\
& =\sum_{i=s}^{k} \sum_{j=0}^{i}\left(\begin{array}{c}
k \\
i
\end{array}\right)\left(\begin{array}{c}
i \\
j
\end{array}\right)(-1)^{j} \beta \int_{0}^{1} z^{\alpha(j+k-i)+\beta-1} d z \\
& =\sum_{i=s}^{k} \sum_{j=0}^{i}\left(\begin{array}{c}
k \\
i
\end{array}\right)\left(\begin{array}{l}
i \\
j
\end{array}\right) \frac{(-1)^{j} \beta}{\alpha(j+k-i)+\beta} .
\end{aligned}
$$

It is interesting to note that the above expression does not depend on the parameter $\theta$. In the following, we compute the maximum likelihood (ML) estimate of $R_{s, k}$. To reach this aim, assume that $x_{1}, x_{2} \ldots, x_{m}$ and $y_{1}, y_{2} \ldots, y_{n}$ are the random samples with the sets of parameters $(\alpha, \theta)$ and $(\beta, \theta)$, respectively. Let $\boldsymbol{\lambda} \equiv(\alpha, \beta, \theta)$, thus the likelihood function based on a given observed sample is

$$
\begin{aligned}
L(\boldsymbol{\lambda})= & \alpha^{m} \beta^{n} \theta^{m+n} \exp \left[-(\theta+1) \sum_{i=1}^{m} \ln \left(1+x_{i}\right)-(\theta+1) \sum_{i=1}^{n} \ln \left(1+y_{i}\right)\right] \\
& \times \exp \left[(\alpha-1) \sum_{i=1}^{m} \ln \left\{1-\left(1+x_{i}\right)^{-\theta}\right\}+(\beta-1) \sum_{i=1}^{n} \ln \left\{1-\left(1+y_{i}\right)^{-\theta}\right\}\right]
\end{aligned}
$$

and the corresponding log-likelihood function is

$$
\begin{aligned}
l(\boldsymbol{\lambda})= & m \ln \alpha+n \ln \beta+(m+n) \ln \theta-(\theta+1)\left[\sum_{i=1}^{m} \ln \left(1+x_{i}\right)+\sum_{i=1}^{n} \ln \left(1+y_{i}\right)\right] \\
& +(\alpha-1) \sum_{i=1}^{m} \ln \left[1-\left(1+x_{i}\right)^{-\theta}\right]+(\beta-1) \sum_{i=1}^{n} \ln \left[1-\left(1+y_{i}\right)^{-\theta}\right] .
\end{aligned}
$$


The MLEs of $\alpha, \beta$, and $\theta$ can be computed as the solution of the following nonlinear equations as

$$
\begin{aligned}
\frac{\partial l(\boldsymbol{\lambda})}{\partial \alpha} & =\frac{m}{\alpha}+\sum_{i=1}^{m} \ln \left[1-\left(1+x_{i}\right)^{-\theta}\right]=0 \\
\frac{\partial l(\boldsymbol{\lambda})}{\partial \beta} & =\frac{n}{\beta}+\sum_{i=1}^{n} \ln \left[1-\left(1+y_{i}\right)^{-\theta}\right]=0 \\
\frac{\partial l(\boldsymbol{\lambda})}{\partial \theta} & =\frac{m+n}{\theta}-\left[\sum_{i=1}^{m} \ln \left(1+x_{i}\right)+\sum_{i=1}^{n} \ln \left(1+y_{i}\right)\right]+(\alpha-1) \sum_{i=1}^{m} \frac{\left(1+x_{i}\right)^{-\theta} \ln \left(1+x_{i}\right)}{1-\left(1+x_{i}\right)^{-\theta}} \\
& +(\beta-1) \sum_{i=1}^{n} \frac{\left(1+y_{i}\right)^{-\theta} \ln \left(1+y_{i}\right)}{1-\left(1+y_{i}\right)^{-\theta}} .
\end{aligned}
$$

We can readily find from Equations (2.4) and (2.5)

$$
\begin{aligned}
& \hat{\alpha}(\theta)=-\frac{m}{\sum_{i=1}^{m} \ln \left[1-\left(1+x_{i}\right)^{-\theta}\right]}, \\
& \hat{\beta}(\theta)=-\frac{n}{\sum_{i=1}^{n} \ln \left[1-\left(1+y_{i}\right)^{-\theta}\right]},
\end{aligned}
$$

and the ML estimate of $\theta$ can be found as the solution of the following nonlinear equation

$$
\begin{aligned}
& \frac{m+n}{\theta}-\left[\sum_{i=1}^{m} \ln \left(1+x_{i}\right)+\sum_{i=1}^{n} \ln \left(1+y_{i}\right)\right]-m \frac{\sum_{i=1}^{m} \frac{\left(1+x_{i}\right)^{-\theta} \ln \left(1+x_{i}\right)}{1-\left(1+x_{i}\right)^{-\theta}}}{\sum_{i=1}^{m} \ln \left[1-\left(1+x_{i}\right)^{-\theta}\right]} \\
& -\sum_{i=1}^{m} \frac{\left(1+x_{i}\right)^{-\theta} \ln \left(1+x_{i}\right)}{1-\left(1+x_{i}\right)^{-\theta}}-n \frac{\sum_{i=1}^{n} \frac{\left(1+y_{i}\right)^{-\theta} \ln \left(1+y_{i}\right)}{1-\left(1+y_{i}\right)^{-\theta}}}{\sum_{i=1}^{n} \ln \left[1-\left(1+y_{i}\right)^{-\theta}\right]}-\sum_{i=1}^{n} \frac{\left(1+y_{i}\right)^{-\theta} \ln \left(1+y_{i}\right)}{1-\left(1+y_{i}\right)^{-\theta}}=0 .
\end{aligned}
$$

Since the ML estimator of $\theta$ cannot be obtained explicitly from the above equation, the Newton-Raphson method or the fixed point method or some other numerical method needs to be used. In this case, we used the fixed point iterative method. By rewriting the above equation, we obtain an equation of the form $f(\theta)=\theta$, where $f(\theta)$ is specified as follows:

$$
\begin{aligned}
f(\theta)=(m+n) & \times\left[\sum_{i=1}^{m} \ln \left(1+x_{i}\right)+\sum_{i=1}^{m} \frac{\left(1+x_{i}\right)^{-\theta} \ln \left(1+x_{i}\right)}{1-\left(1+x_{i}\right)^{-\theta}}+m \frac{\sum_{i=1}^{m} \frac{\left(1+x_{i}\right)^{-\theta} \ln \left(1+x_{i}\right)}{1-\left(1+x_{i}\right)^{-\theta}}}{\sum_{i=1}^{m} \ln \left[1-\left(1+x_{i}\right)^{-\theta}\right]}\right. \\
& \left.+\sum_{i=1}^{n} \ln \left(1+y_{i}\right)+\sum_{i=1}^{n} \frac{\left(1+y_{i}\right)^{-\theta} \ln \left(1+y_{i}\right)}{1-\left(1+y_{i}\right)^{-\theta}}+n \frac{\sum_{i=1}^{n} \frac{\left(1+y_{i}\right)^{-\theta} \ln \left(1+y_{i}\right)}{1-\left(1+y_{i}\right)^{-\theta}}}{\sum_{i=1}^{n} \ln \left[1-\left(1+y_{i}\right)^{-\theta}\right]}\right],
\end{aligned}
$$

and $\hat{\theta}$ can be obtained by using a simple iterative procedure as $f\left(\theta_{j}\right)=\theta_{j+1}$. Here, $\theta_{j}$ is the jth iteration of $\hat{\theta}_{j}$. The iteration procedure should be stopped when $\left|\theta_{j}-\theta_{j+1}\right|$ is sufficiently small. When $\hat{\theta}$ is determined, the ML estimators of $\alpha$ and $\beta$ can be obtained from Equations (2.7) and (2.8), respectively. To compute the ML estimate of $\theta$, we have used the true value of $\theta$ as the initial guess value. In the real data application described, 
later on, we obtained the initial value of $\theta$ by drawing the two functions $y=\theta$ and $y=f(\theta)$ (given in Equation (2.9)) and observing their intersection. After calculating $\alpha, \beta$, and $\theta$, as well as the invariant property of MLE, the MLE of $R_{s, k}$ is obtained as

$$
\hat{R}_{s, k}^{M L E}=\sum_{i=s}^{k} \sum_{j=0}^{i}\left(\begin{array}{l}
k \\
i
\end{array}\right)\left(\begin{array}{l}
i \\
j
\end{array}\right) \frac{(-1)^{j} \hat{\beta}}{\hat{\alpha}(j+k-i)+\hat{\beta}} .
$$

It should be noted that to compute the MLEs of $\alpha, \beta$, and $\theta$, one can apply the existing $\mathrm{R}$ functions such as nlm, nlminb, optim and so on. The advantage of these functions is that they permit the direct maximization of the log-likelihood equation and do not need partial derivatives of the log-likelihood equation. For the sake of comparison with the fixed point iterative method, we also used the nlminb function to determine the MLEs of $\alpha, \beta$, and $\theta$. We reached exactly the same results. For this reason, and also for the sake of brevity, we did not include the results in the simulation section. However, the $\mathrm{R}$ codes based on the fixed point method and nlminb function have been provided in Appendix A and $\mathrm{B}$, respectively.

The existence and uniqueness of the MLEs is one of the important topics in statistical lectures. Since the likelihood equations are not analytically tractable, we use the superimposed curves of log-likelihood equations to show the existence and uniqueness of the MLEs. In this regard, we apply the method of [45]. The necessary steps to achieve this goal are as follows:

Step 1: We generate independent random samples of sizes 10 from $X \sim I K u(1,5)$ and $Y \sim I K u(3,5),(\alpha=1, \beta=3$ and $\theta=5)$. The observations for strength data are as follows; $0.29,0.05,1.14,0.67,0.02,0.08,0.19,0.20,0.02,0.29$. Also, the observations for stress data are as follows; $0.26,0.27,0.62,0.72,0.55,0.19,0.09,0.35,0.20,1.26$.

Step 2: The plots of the $\log$-likelihood equations of $\alpha\left(\frac{d \log L}{d \alpha}=0\right)$ and $\beta\left(\frac{d \log L}{d \beta}=0\right)$ are drawn. Also, the plot of Equation (2.6) is sketched in terms of $\alpha$ and $\beta$ while one of them is kept as fixed at a time. The result is shown in Figure 3.

Step 3: Based on Figure 3, it is readily apparent that there exists one intersection point $(\hat{\alpha}=1.0810, \hat{\beta}=2.9211$, and $\hat{\theta}=4.9682)$ which confirms that the solution of the log-likelihood equations exist and are unique.

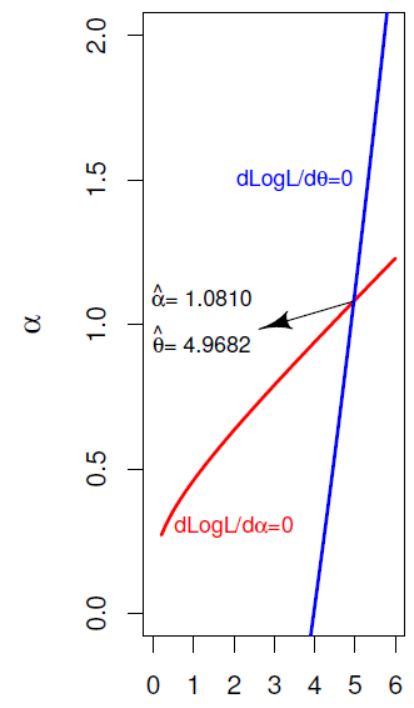

$\theta$

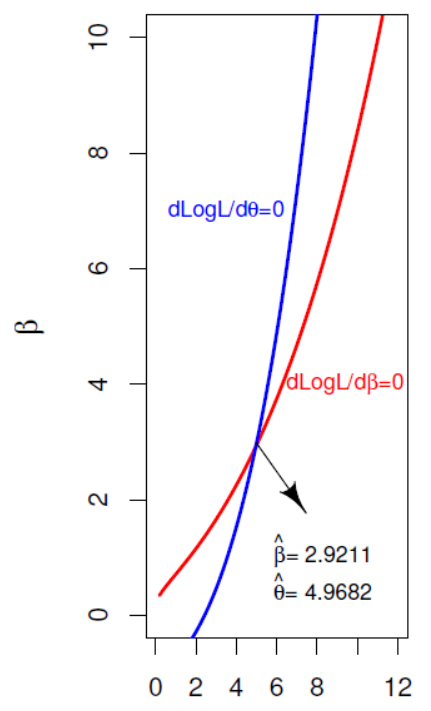

$\theta$

Figure 3. Plots of log-likelihood equations and their intersection points. 
Step 4: The plots of profile log-likelihood equation in terms of parameters $\alpha, \beta$, and $\theta$ are sketched in Figure 4. Based on Figure 4, it can be seen that the intersection point maximizes the log-likelihood equation of the parameters.

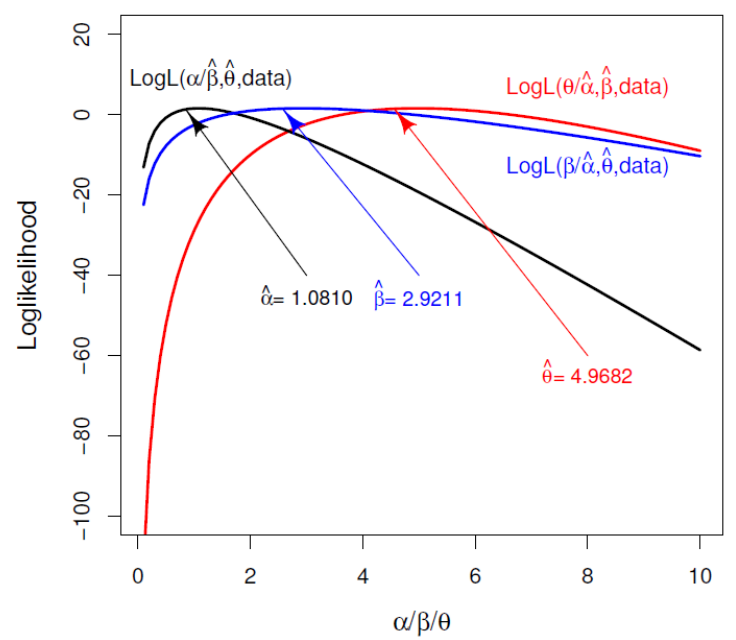

Figure 4. Log-likelihood profile of the parameters $\alpha, \beta$, and $\theta$ along with MLEs.

The above process is also used for different combinations of parameters as well as different sample sizes and it was observed that the ML estimates of parameters exist and are unique.

Now, considering $\boldsymbol{\lambda} \equiv(\alpha, \beta, \theta)$, we determine the ACI of $R_{s, k}$ using the asymptotic distribution of $\boldsymbol{\lambda}$ and delta method. The expected Fisher information matrix of $\boldsymbol{\lambda}$ is specified as follows:

$$
I(\boldsymbol{\lambda})=E\left[\begin{array}{ccc}
-\frac{\partial^{2} l}{\partial \alpha^{2}} & -\frac{\partial^{2} l}{\partial \alpha \partial \beta} & -\frac{\partial^{2} l}{\partial \alpha \partial \theta} \\
-\frac{\partial^{2} l}{\partial \beta \partial \alpha} & -\frac{\partial^{2} l}{\partial \beta^{2}} & -\frac{\partial^{2} l}{\partial \beta \partial \theta} \\
-\frac{\partial^{2} l}{\partial \theta \partial \alpha} & -\frac{\partial^{2} l}{\partial \theta \partial \beta} & -\frac{\partial^{2} l}{\partial \theta^{2}}
\end{array}\right]=E(A)
$$

where

$$
\begin{aligned}
& a_{11}=\frac{m}{\alpha^{2}}, \quad a_{12}=a_{21}=0, \quad a_{22}=\frac{n}{\beta^{2}}, \quad a_{13}=a_{31}=-\sum_{i=1}^{m} \frac{\left(1+x_{i}\right)^{-\theta} \ln \left(1+x_{i}\right)}{1-\left(1+x_{i}\right)^{-\theta}}, \\
& a_{23}=a_{32}=-\sum_{i=1}^{n} \frac{\left(1+y_{i}\right)^{-\theta} \ln \left(1+y_{i}\right)}{1-\left(1+y_{i}\right)^{-\theta}}, \\
& a_{33}=\frac{m+n}{\theta^{2}}+(\alpha-1) \sum_{i=1}^{m} \frac{\left(1+x_{i}\right)^{-\theta} \ln ^{2}\left(1+x_{i}\right)}{\left[1-\left(1+x_{i}\right)^{-\theta}\right]^{2}}+(\beta-1) \sum_{i=1}^{n} \frac{\left(1+y_{i}\right)^{-\theta} \ln ^{2}\left(1+y_{i}\right)}{\left[1-\left(1+y_{i}\right)^{-\theta}\right]^{2}} .
\end{aligned}
$$

Unfortunately, it is very difficult to determine the expectation of the above expressions analytically. Thus, we used the observed information matrix $A$ as a consistent estimator of $I(\boldsymbol{\lambda})$ by omitting the expectation operator $E$. The MLE of $R_{s, k}$ has an asymptotically normal distribution with the mean $R_{s, k}$ and variance

$$
H_{s, k}=\sum_{i=1}^{3} \sum_{j=1}^{3} \frac{\partial R_{s, k}}{\partial \lambda_{i}} \frac{\partial R_{s, k}}{\partial \lambda_{j}} A_{i j}^{-1}
$$


where $A_{i j}^{-1}$ is the $(i, j)$ th element of the inverse of $A$. Also, we have

$$
\begin{aligned}
& \frac{\partial R_{s, k}}{\partial \alpha}=\sum_{i=s}^{k} \sum_{j=0}^{i}\left(\begin{array}{c}
k \\
i
\end{array}\right)\left(\begin{array}{l}
i \\
j
\end{array}\right) \frac{(-1)^{j+1} \beta(j+k-i)}{[\alpha(j+k-i)+\beta]^{2}}, \\
& \frac{\partial R_{s, k}}{\partial \beta}=\sum_{i=s}^{k} \sum_{j=0}^{i}\left(\begin{array}{c}
k \\
i
\end{array}\right)\left(\begin{array}{l}
i \\
j
\end{array}\right) \frac{(-1)^{j} \alpha(j+k-i)}{[\alpha(j+k-i)+\beta]^{2}} .
\end{aligned}
$$

Hence, the asymptotic variance is given by

$$
\hat{H}_{s, k}=A_{11}^{-1}\left(\frac{\partial R_{s, k}}{\partial \alpha}\right)^{2}+2 A_{12}^{-1} \frac{\partial R_{s, k}}{\partial \alpha} \frac{\partial R_{s, k}}{\partial \beta}+A_{22}^{-1}\left(\frac{\partial R_{s, k}}{\partial \beta}\right)^{2} \mid(\hat{\alpha}, \hat{\beta})
$$

and the $100(1-\delta) \%$ ACI of $R_{s, k}$ is constructed as

$$
\hat{R}_{s, k}^{M L E} \pm z_{\delta / 2} \sqrt{\hat{H}_{s, k}}
$$

where, $z_{\delta / 2}$ is the upper $\delta / 2$ th quantile of the $N(0,1)$.

It should be pointed out that, the confidence interval obtained from the above equation may not be within the interval $(0,1)$. In this situation, we follow the methods of [19] and [35] and use the Logit and Arcsin transformations for $R_{s, k}$ as $g\left(R_{s, k}\right)=$ $\log \left[R_{s, k} /\left(1-R_{s, k}\right)\right]$ and $h\left(R_{s, k}\right)=\operatorname{Arcsin}\left(\sqrt{R_{s, k}}\right)$, respectively. Based on these transformations, the $100(1-\delta) \% \mathrm{ACI}$ for $g\left(R_{s, k}\right)$ and $R_{s, k}$, respectively, take the following form:

and

$$
\log \left(\frac{R_{s, k}}{1-R_{s, k}}\right) \pm z_{\delta / 2} \frac{\sqrt{\hat{H}_{s, k}}}{\hat{R}_{s, k} \sqrt{\left(1-\hat{R}_{s, k}\right)}} \equiv\left(L_{1}, U_{1}\right)
$$

$$
\left(\frac{e^{L_{1}}}{1+e^{L_{1}}}, \frac{e^{U_{1}}}{1+e^{U_{1}}}\right)
$$

Also, the $100(1-\delta) \%$ ACI for $h\left(R_{s, k}\right)$ and $R_{s, k}$, respectively, are as follows:

and

$$
\operatorname{Arcsin}\left(\sqrt{\hat{R}_{s, k}}\right) \pm z_{\delta / 2} \frac{\sqrt{\hat{H}_{s, k}}}{2 \sqrt{\hat{R}_{s, k}\left(1-\hat{R}_{s, k}\right)}} \equiv\left(L_{2}, U_{2}\right)
$$

$$
\left(\sin ^{2} L_{2}, \sin ^{2} U_{2}\right)
$$

where, $z_{\delta / 2}$ is the upper $\delta / 2$ th quantile of the $N(0,1)$. Hereafter, we denote AST, LOGT and NT for ACIs which are obtained respectively based on Arcsin transformation, Logit transformation and not using either of these two transformations.

\subsection{Bayes estimation of $\boldsymbol{R}_{s, k}$}

In this section, we derive the Bayes estimate and corresponding HPD credible interval of $R_{s, k}$ under the SE loss function. To achieve this aim, we suppose that the independent random variables $\alpha, \beta$, and $\theta$ have Gamma priors with positive parameters $\left(a_{1}, b_{1}\right),\left(a_{2}, b_{2}\right)$, and $\left(a_{3}, b_{3}\right)$, respectively. Based on the observations, the joint posterior density function is

$$
\begin{aligned}
\pi(\alpha, \beta, \theta \mid \mathbf{x}, \mathbf{y}) & =\frac{L(\mathbf{x}, \mathbf{y} \mid \alpha, \beta, \theta) \pi_{1}(\alpha) \pi_{2}(\beta) \pi_{3}(\theta)}{\int_{0}^{\infty} \int_{0}^{\infty} \int_{0}^{\infty} L(\mathbf{x}, \mathbf{y} \mid \alpha, \beta, \theta) \pi_{1}(\alpha) \pi_{2}(\beta) \pi_{3}(\theta) d \alpha d \beta d \theta} \\
& \propto \alpha^{m+a_{1}-1} \beta^{n+a_{2}-1} \theta^{m+n+a_{3}-1} \exp \left[-\alpha\left(b_{1}+U_{\theta}\right)-\beta\left(b_{2}+V_{\theta}\right)+W_{\theta}\right]
\end{aligned}
$$


where

$$
\begin{gathered}
U_{\theta}=-\sum_{i=1}^{m} \ln \left[1-\left(1+x_{i}\right)^{-\theta}\right], \quad V(\theta)=-\sum_{i=1}^{n} \ln \left[1-\left(1+y_{i}\right)^{-\theta}\right], \\
W_{\theta}=U_{\theta}+V_{\theta}-\theta\left[b_{3}+\sum_{i=1}^{m} \ln \left(1+x_{i}\right)+\sum_{i=1}^{n} \ln \left(1+y_{i}\right)\right] .
\end{gathered}
$$

Then, the Bayes estimate of $R_{s, k}$, against the SE loss function is calculated by

$$
\hat{R}_{s, k}^{\text {Bayes }}=E\left(R_{s, k} \mid \mathbf{x}, \mathbf{y}\right)=\int_{0}^{\infty} \int_{0}^{\infty} \int_{0}^{\infty} R_{s, k} \pi(\alpha, \beta, \theta \mid \mathbf{x}, \mathbf{y}) d \alpha d \beta d \theta .
$$

Since the Bayes estimate of $R_{s, k}$ cannot be computed analytically, therefore we apply the MCMC technique. From Equation (2.12), the posterior pdfs of $\alpha, \beta$, and $\theta$ are as follows, respectively:

$$
\begin{aligned}
& \alpha \mid \theta, \mathbf{x}, \mathbf{y} \sim \operatorname{Gamma}\left[a_{1}+m, b_{1}+U_{\theta}\right] \\
& \beta \mid \theta, \mathbf{x}, \mathbf{y} \sim \operatorname{Gamma}\left[a_{2}+n, b_{2}+V_{\theta}\right] \\
& \pi(\theta \mid \alpha, \beta, \mathbf{x}, \mathbf{y}) \propto \theta^{m+n+a_{3}-1} \exp \left[-\alpha U_{\theta}-\beta V_{\theta}+W_{\theta}\right] .
\end{aligned}
$$

We see that the posterior pdfs of $\alpha$ and $\beta$ given in Equations (2.13) and (2.14) have Gamma distributions. Thus, using the Gibbs sampling method, we generate random samples from $\alpha$ and $\beta$. On the other side, the posterior pdf of $\theta$ given in Equation (2.15) does not reduce analytically to a known distribution. In this situation, we follow the method of [17]. If a posterior density function is unimodal and roughly symmetric, therefore it is generally appropriate to approximate it by a normal distribution. The correctness of this is confirmed by Figure 5 which gives the plot of the posterior density of $\theta$ for different combinations of parameters $(\alpha, \beta)$. Hence, we apply the Metropolis-Hastings technique to generate a sample for $\theta$ by considering a normal proposal distribution. The necessary steps to achieve this goal are as follows:

Step 1: Start with an initial conjecture $\left(\alpha^{(0)}, \beta^{(0)}, \theta^{(0)}\right)$.

Step 2: Set $l=1$.

Step 3: Generate $\alpha^{(l)}$ from $\operatorname{Gamma}\left(m+a_{1}, b_{1}+U_{\theta^{(l-1)}}\right)$.

Step 4: Generate $\beta^{(l)}$ from $\operatorname{Gamma}\left(n+a_{2}, b_{2}+V_{\theta^{(l-1)}}\right)$.

Step 5: Generate $\theta^{(l)}$ from $\pi\left(\theta \mid \alpha^{(l-1)}, \beta^{(l-1)}, \mathbf{x}, \mathbf{y}\right)$ using the Metropolis-Hastings with proposal distribution $N\left(\theta^{(l-1)}, H\right)$, where $H$ can be computed from Equation (2.11).

Step 6: Compute $R_{s, k}^{(l)}=\sum_{i=s}^{k} \sum_{j=0}^{i}\left(\begin{array}{c}k \\ i\end{array}\right)\left(\begin{array}{l}i \\ j\end{array}\right) \frac{(-1)^{j} \beta^{(l)}}{\alpha^{(l)}(j+k-i)+\beta^{(l)}}$.

Step 7: Set $l=l+1$.

Step 8: Repeat Steps 3-7, $N$ times, and determine $R_{s, k}^{(l)}$ for $l=1,2, \ldots, N$.

The Bayes estimate of $R_{s, k}$, based on the MCMC method, is calculated by

$$
\hat{R}_{s, k}^{M C}=\frac{1}{N-N_{0}} \sum_{l=N_{0}+1}^{N} R_{s, k}^{(l)},
$$

where $N_{0}$ is the burn-in period. Also, the $100(1-\delta) \%$ HPD credible interval for $R_{s, k}$ can be computed by using the method of [13], by minimizing

$$
\left(R_{s, k}\left(\left[(1-\delta)\left(N-N_{0}\right)\right]+i\right)-R_{s, k}{ }^{(i)}\right), \quad 1 \leq i \leq \delta\left(N-N_{0}\right),
$$

where [.] denotes the largest integer function and the values of $R_{s, k}$ are ranked in ascending order from 1 to $\left(N-N_{0}\right)$. 

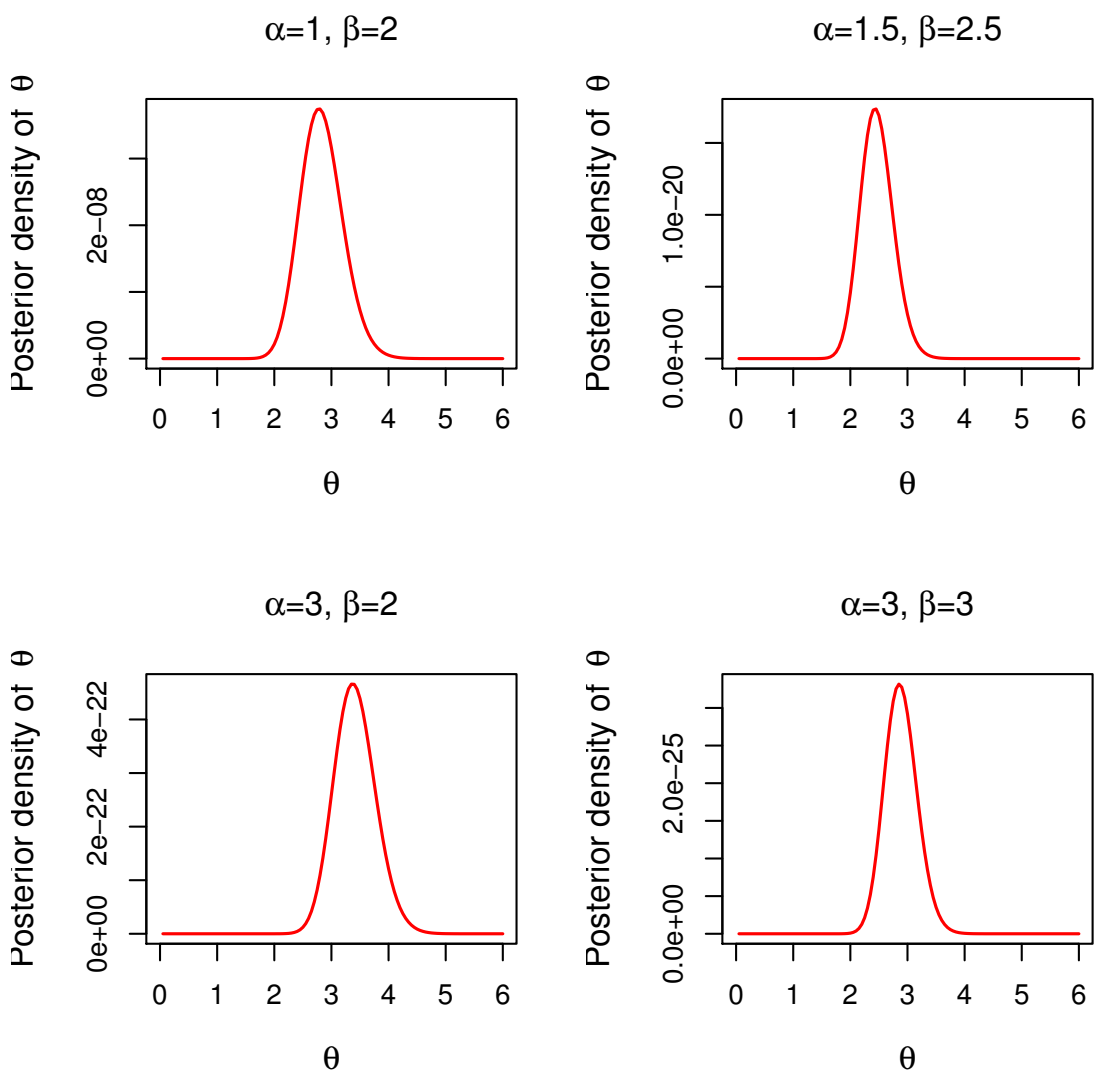

Figure 5. The posterior density of the parameter $\theta$ for different combinations of $(\alpha, \beta)$.

\section{Estimation of $\boldsymbol{R}_{s, k}$ when $\boldsymbol{\theta}$ is known}

\subsection{MLE of $\boldsymbol{R}_{s, k}$}

Assume that $x_{1}, x_{2} \ldots, x_{m}$ and $y_{1}, y_{2} \ldots, y_{n}$ are the random samples from the IKu distribution with the sets of parameters $\left(\alpha, \theta_{0}\right)$ and $\left(\beta, \theta_{0}\right)$, respectively. Thus the log-likelihood function based on a given observed sample is

$$
l\left(\alpha, \beta \mid \theta_{0}, \mathbf{x}, \mathbf{y}\right)=m \ln \alpha+n \ln \beta+\alpha \sum_{i=1}^{m} \ln \left[1-\left(1+x_{i}\right)^{-\theta_{0}}\right]+\beta \sum_{i=1}^{n} \ln \left[1-\left(1+y_{i}\right)^{-\theta_{0}}\right],
$$

where the constant terms are omitted from the above equation. From Equation (3.1), it is easily seen that the MLEs of $\alpha$ and $\beta$ are given as

$$
\hat{\alpha}=-\frac{m}{\sum_{i=1}^{m} \ln \left[1-\left(1+x_{i}\right)^{-\theta_{0}}\right]}, \quad \hat{\beta}=-\frac{n}{\sum_{i=1}^{n} \ln \left[1-\left(1+y_{i}\right)^{-\theta_{0}}\right]} .
$$

Therefore, by the invariant property of MLE, the MLE of $R_{s, k}$ is obtained from Equation (2.1). Proceeding in a way similar to Section 2.1, the MLE of $R_{s, k}$ has an asymptotically normal distribution with the mean $R_{s, k}$ and variance

$$
\hat{H}_{s, k}=\frac{\alpha^{2}}{m}\left(\frac{\partial R_{s, k}}{\partial \alpha}\right)^{2}+\frac{\beta^{2}}{n}\left(\frac{\partial R_{s, k}}{\partial \beta}\right)^{2} \mid(\hat{\alpha}, \hat{\beta}) .
$$

Hence, the $100(1-\delta) \%$ ACI of $R_{s, k}$ is constructed as

$$
\hat{R}_{s, k}^{M L E} \pm z_{\delta / 2} \sqrt{\hat{H}_{s, k}}
$$




\subsection{UMVUE of $\boldsymbol{R}_{s, k}$}

In this subsection, we derive the UMVUE of $R_{s, k}$ through an unbiased estimator of $\varphi(\alpha, \beta)=(-1)^{j} \beta /[\alpha(j+k-i)+\beta]$ and a complete sufficient statistic of $(\alpha, \beta)$. It can be seen that from Equation $(2.2),\left(U^{*}, V^{*}\right)$ is the complete sufficient statistic of $(\alpha, \beta)$, where

$$
U^{*}=-\sum_{i=1}^{m} \ln \left[1-\left(1+x_{i}\right)^{-\theta_{0}}\right], \quad V^{*}=\sum_{i=1}^{n} \ln \left[1-\left(1+y_{i}\right)^{-\theta_{0}}\right] .
$$

Furthermore, the statistic $U^{*}$ has Gamma distribution with parameters $m$ and $\alpha$, and the statistic $V^{*}$ has Gamma distribution with parameters $n$ and $\beta$. Let

$$
U^{0}=-\ln \left[1-\left(1+X_{1}\right)^{-\theta_{0}}\right], \quad V^{0}=-\ln \left[1-\left(1+Y_{1}\right)^{-\theta_{0}}\right] .
$$

It is easy to know that $U^{0}$ and $V^{0}$ come from the exponential distributions with parameters $\alpha$ and $\beta$, respectively. Hence,

$$
\psi\left(U^{0}, V^{0}\right)= \begin{cases}1, & U^{0}>(j+k-i) V^{0} \\ 0, & \text { otherwise, }\end{cases}
$$

is an unbiased estimator of $\varphi(\alpha, \beta)$. Applying Lehmann's theorem, the UMVUE of $\varphi(\alpha, \beta)$ is specified by

$$
\begin{aligned}
\hat{\varphi}_{U M}(\alpha, \beta) & =E\left[\psi\left(U^{0}, V^{0}\right) \mid U^{*}=u^{*}, V^{*}=v^{*}\right] \\
& =\int A \int f_{U^{0} \mid U^{*}=u^{*}}\left(u^{0} \mid u^{*}\right) f_{V^{0} \mid V^{*}=v^{*}}\left(v^{0} \mid v^{*}\right) d u^{0} d v^{0},
\end{aligned}
$$

where $A=\left\{\left(u^{0}, v^{0}\right): 0<u^{0}<u^{*}, 0<v^{0}<v^{*}, u^{0}>(j+k-i) v^{0}\right\}$. This integral can be discussed with regards to $h<1$ and $h>1$, where $h=(j+k-i) v^{*} / u^{*}$. When $h<1$, the integral in Equation (3.3) reduces to

$$
\begin{aligned}
\hat{\varphi}_{U M}(\alpha, \beta) & =\int_{0}^{v^{*}} \int_{(j+k-i) v^{0}}^{u^{*}} \frac{(m-1)(n-1)}{u^{*} v^{*}}\left(1-\frac{u^{0}}{u^{*}}\right)^{m-2}\left(1-\frac{v^{0}}{v^{*}}\right)^{n-2} d u^{0} d v^{0} \\
& =(n-1) \int_{0}^{1}(1-z) \quad(1-h z)^{m-1} d z, \quad \text { where } z=v^{0} / v^{*} \\
& =\sum_{l=0}^{m-1}(-1)^{l}(h)^{l}\left(\begin{array}{c}
m-1 \\
l
\end{array}\right) /\left(\begin{array}{c}
n+l-1 \\
l
\end{array}\right) .
\end{aligned}
$$

Similarly, when $h>1$, the integral Equation (3.3) reduces to

$$
\begin{aligned}
\hat{\varphi}_{U M}(\alpha, \beta) & =\int_{0}^{u^{*}} \int_{0}^{u^{0} /(j+k-i)} \frac{(m-1)(n-1)}{u^{*} v^{*}}\left(1-\frac{u^{0}}{u^{*}}\right)^{m-2}\left(1-\frac{v^{0}}{v^{*}}\right)^{n-2} d v^{0} d u^{0} \\
& =1-(m-1) \int_{0}^{1}(1-z)^{m-2}\left(1-h^{-1} z\right)^{n-1} d z, \quad \text { where } z=u^{0} / u^{*} \\
& =1-\sum_{l=0}^{n-1}(-1)^{l}(h)^{-l}\left(\begin{array}{c}
n-1 \\
l
\end{array}\right) /\left(\begin{array}{c}
m+l-1 \\
l
\end{array}\right) .
\end{aligned}
$$

Thus, the $\hat{\varphi}_{U M}(\alpha, \beta)$ is obtained from Equations (3.4) and (3.5). Finally, the UMVUE of $R_{s, k}$ is determined by applying the linearity property of UMVUE as follows:

$$
\hat{R}_{s, k}^{U M}=\sum_{i=s}^{k} \sum_{j=0}^{k}\left(\begin{array}{c}
k \\
i
\end{array}\right)\left(\begin{array}{c}
k \\
j
\end{array}\right)(-1)^{j} \hat{\varphi}_{U M}(\alpha, \beta) .
$$




\subsection{Bayes estimation of $\boldsymbol{R}_{s, k}$}

In this subsection, we derive the Bayes estimate of $R_{s, k}$ along with its HPD credible interval under the SE loss function. To achieve this aim, we suppose that the independent random variables $\alpha$ and $\beta$ have Gamma priors with positive parameters $\left(a_{1}, b_{1}\right)$ and $\left(a_{2}, b_{2}\right)$, respectively. Based on the observations, the joint posterior density function is

$$
\begin{aligned}
& \pi(\alpha, \beta \mid \mathbf{x}, \mathbf{y})=\frac{L(\mathbf{x}, \mathbf{y} \mid \alpha, \beta) \pi_{1}(\alpha) \pi_{2}(\beta)}{\int_{0}^{\infty} \int_{0}^{\infty} L(\mathbf{x}, \mathbf{y} \mid \alpha, \beta) \pi_{1}(\alpha) \pi_{2}(\beta) d \alpha d \beta} \\
& \quad=\frac{\left(b_{1}+U^{*}\right)^{m+a_{1}}\left(b_{2}+V^{*}\right)^{n+a_{2}}}{\Gamma\left(m+a_{1}\right) \Gamma\left(n+a_{2}\right)} \alpha^{m+a_{1}-1} \beta^{n+a_{2}-1} \exp \left[-\alpha\left(b_{1}+U^{*}\right)-\beta\left(b_{2}+V^{*}\right)\right],
\end{aligned}
$$

where $U^{*}$ and $V^{*}$ are defined in Equation (3.2). Then, the Bayes estimate of $R_{s, k}$ is calculated by

$$
\hat{R}_{s, k}^{\text {Bayes }}=E\left(R_{s, k} \mid \mathbf{x}, \mathbf{y}\right)=\sum_{i=s}^{k} \sum_{j=0}^{i}\left(\begin{array}{c}
k \\
i
\end{array}\right)\left(\begin{array}{c}
i \\
j
\end{array}\right)(-1)^{j} \int_{0}^{\infty} \int_{0}^{\infty} \frac{\beta}{\alpha(j+k-i)+\beta} \pi(\alpha, \beta \mid \mathbf{x}, \mathbf{y}) d \alpha d \beta .
$$

Now, using the results of [25], the Bayes estimate of $R_{s, k}$ can be rewritten as

$$
\hat{R}_{s, k}^{\text {Bayes }}= \begin{cases}\sum_{i=s}^{k} \sum_{j=0}^{k}\left(\begin{array}{c}
k \\
i
\end{array}\right)\left(\begin{array}{c}
k \\
j
\end{array}\right)(-1)^{j}(1-w)^{n+a_{2}} \frac{n+a_{2}}{q}{ }_{1} F_{2}\left(q, n+a_{2}+1 ; q+1, w\right), & |w|<1 \\
\sum_{i=s}^{k} \sum_{j=0}^{k}\left(\begin{array}{c}
k \\
i
\end{array}\right)\left(\begin{array}{c}
k \\
j
\end{array}\right) \frac{(-1)^{j}\left(n+a_{2}\right)}{q(1-w)^{m+a_{1}} 1} F_{2}\left(q, m+a_{1}+1 ; q+1, \frac{w}{w-1}\right), & w<-1\end{cases}
$$

where $q=m+n+a_{1}+a_{2}$ and $w=1-\frac{\left(b_{2}+V^{*}\right)(i+j)}{b_{1}+U^{*}}$. Notice that

$$
{ }_{2} F_{1}(a, b ; c, x)=\frac{1}{\operatorname{Beta}(a, c-a)} \int_{0}^{1} w^{a-1}(1-w)^{c-a-1}(1-x w)^{-b} d w, \quad|w|<1,
$$

is the hypergeometric function that can be obtained using $\mathrm{R}$ software command hyperg_2F1() from ' $g s l$ ' package. Also, the $100(1-\delta) \%$ HPD credible interval for $R_{s, k}$ can be computed by using the method of [13] proceeding in a way similar to Section 2 .

\section{Simulation study}

In this section, we perform Monte Carlo simulations to compare the performances of different estimates of $R_{s, k}$ by using the classical and Bayesian methods. In this regard, we generate random samples from the stress and strength variables for $(s, k)=(2,5)$ and for different sample sizes 10,30, and 50. The criteria of mean square error (MSE), average length (AL) as well as coverage probability $(\mathrm{CP})$ at confidence level of $95 \%$, are used to evaluate the simulation results. All of the computations are done by using R 3.4.4 based on 10,000 replications.

To study the Bayes estimation, two schemes for hyperparameters are considered. We investigate the hyperparameters $\left(a_{i}, b_{i}\right)=(0.0001,0.0001), i=1,2,3$ under the noninformative prior. In the case of informative prior, we followed the idea of [47], such that priors means are taken as the true values of the parameters and priors variances are little (equal to 1). We use Prior 1 and Prior 2 to denote the Bayes estimators based on noninformative and informative priors, respectively. Tables 1 and 2 represent the details of the simulations when $\theta$ is unknown and known, respectively. 
Table 1. Different combinations of stress and strength parameters along with the true values of $R_{2,5}$ as well as different priors for Monte Carlo simulations ( $\theta$ is unknown).

\begin{tabular}{ccccccc}
\hline & & \multicolumn{2}{c}{ Prior 1 } & & \multicolumn{3}{c}{ Prior 2 } \\
\cline { 6 - 7 } \cline { 5 - 6 }$(\alpha, \beta, \theta)$ & $R_{2,5}$ & $\left(a_{i}, b_{i}\right), i=1,2,3$ & & $\left(a_{1}, b_{1}\right)$ & $\left(a_{2}, b_{2}\right)$ & $\left(a_{3}, b_{3}\right)$ \\
\hline$(0.5,4.8253,1)$ & 0.1 & $(0.0001,0.0001)$ & & $(0.25,0.5)$ & $(23.2835,4.8253)$ & $(1,1)$ \\
$(0.5,2.7563,1.5)$ & 0.2 & $(0.0001,0.0001)$ & & $(0.25,0.5)$ & $(7.5972,2.7563)$ & $(2.25,1.5)$ \\
$(0.5,1.8401,1.5)$ & 0.3 & $(0.0001,0.0001)$ & & $(0.25,0.5)$ & $(3.3860,1.8401)$ & $(2.25,1.5)$ \\
$(0.5,1.2943,1.5)$ & 0.4 & $(0.0001,0.0001)$ & & $(0.25,0.5)$ & $(1.6752,1.2943)$ & $(2.25,1.5)$ \\
$(1,1.8422,2)$ & 0.5 & $(0.0001,0.0001)$ & & $(1,1)$ & $(3.3937,1.8422)$ & $(4,2)$ \\
$(1,1.2950,2)$ & 0.6 & $(0.0001,0.0001)$ & & $(1,1)$ & $(1.6770,1.2950)$ & $(4,2)$ \\
$(1.5,1.3082,2)$ & 0.7 & $(0.0001,0.0001)$ & & $(2.25,1.5)$ & $(1.7114,1.3082)$ & $(4,2)$ \\
$(2.5,1.3123,2)$ & 0.8 & $(0.0001,0.0001)$ & & $(6.25,2.5)$ & $(1.7221,1.3123)$ & $(4,2)$ \\
$(3,0.7214,1.5)$ & 0.9 & $(0.0001,0.0001)$ & $(9,3)$ & $(0.5204,0.7214)$ & $(2.25,1.5)$ \\
\hline
\end{tabular}

Table 2. Different combinations of stress and strength parameters along with the true values of $R_{2,5}$ as well as different priors for Monte Carlo simulations $(\theta$ is known equal to 5 ).

\begin{tabular}{cccccc}
\hline & & Prior 1 & & \multicolumn{2}{c}{ Prior 2 } \\
\cline { 3 - 3 } \cline { 5 - 6 }$(\alpha, \beta)$ & $R_{2,5}$ & $\left(a_{i}, b_{i}\right), i=1,2$ & & $\left(a_{1}, b_{1}\right)$ & $\left(a_{2}, b_{2}\right)$ \\
\hline$(2,19.3019)$ & 0.1 & $(0.0001,0.0001)$ & & $(4,2)$ & $(372.5633,19.3019)$ \\
$(2,11.0249)$ & 0.2 & $(0.0001,0.0001)$ & & $(4,2)$ & $(121.5484,11.0249)$ \\
$(2,7.3606)$ & 0.3 & $(0.0001,0.0001)$ & & $(4,2)$ & $(54.1784,7.3606)$ \\
$(2,5.1774)$ & 0.4 & $(0.0001,0.0001)$ & & $(4,2)$ & $(26.8055,5.1774)$ \\
$(3,5.5328)$ & 0.5 & $(0.0001,0.0001)$ & & $(9,3)$ & $(30.6119,5.5328)$ \\
$(3,3.8853)$ & 0.6 & $(0.0001,0.0001)$ & & $(9,3)$ & $(15.0956,3.8853)$ \\
$(4,3.4743)$ & 0.7 & $(0.0001,0.0001)$ & & $(16,4)$ & $(12.0708,3.4743)$ \\
$(5,2.6246)$ & 0.8 & $(0.0001,0.0001)$ & & $(25,5)$ & $(6.8885,2.6246)$ \\
$(7,1.6834)$ & 0.9 & $(0.0001,0.0001)$ & $(49,7)$ & $(2.8338,1.6834)$ \\
\hline
\end{tabular}

To conduct the Metropolis-Hastings technique, Markov chains of size 70000 are generated and the first 20000 of the samples are discarded to diminish the effect of the starting distribution. Then, for the sake to reduce the dependence between the generated samples, we take every 5 th sampled value. To check the convergence of MCMC simulations, we are computed the scale reduction factor estimate. This estimate is obtained by $\sqrt{\operatorname{Var}(\Omega) / P}$, where $\Omega$ is the estimate of interest and $\operatorname{Var}(\Omega)=(n-1) P / n+Q / n$. In this formula, $n$ represents the iteration number of each chain, $P$ represents the within-sequence variance and $Q$ represents the between-sequence variance. It is found that the values of the scale factors for MCMC estimators are lower than 1.1 which are appropriate values for their convergence. All of the results are reported in Tables 3-6. Based on these tables, the following findings can be drawn.

- The MSEs of all the estimators decrease with increasing sample sizes, as anticipated.

- The MSEs of all the estimators are maximized when $R_{s, k}$ is 0.5 and they become smaller and smaller as we go away to extreme values.

- In the case where $\theta$ is known, the Bayes estimator based on Prior 2 has a smaller MSE than other estimators. Also, there is no difference between the Bayes estimator based on Prior 1, MLE, and UMVUE except for $n=10$. In this sample size, in most cases, the ordering of performance is the Bayes estimator based on 
Prior 1, MLE, and UMVUE. However, for extreme values of $R_{s, k}$, the opposite of this ordering is observed.

Table 3. Point estimates of $R_{2,5}$ along with MSEs (presented in parenthesis) when $\theta$ is unknown.

\begin{tabular}{|c|c|c|c|c|c|}
\hline \multirow[b]{2}{*}{$(\alpha, \beta, \theta)$} & \multirow[b]{2}{*}{$R_{2,5}$} & \multirow[b]{2}{*}{$n$} & \multirow[b]{2}{*}{ MLE } & \multicolumn{2}{|c|}{ Bayesian } \\
\hline & & & & Prior 1 & Prior 2 \\
\hline \multirow{3}{*}{$(0.5,4.8253,1)$} & \multirow{3}{*}{0.1} & 10 & $0.0988(0.0055)$ & $0.1300(0.0061)$ & $0.1143(0.0025)$ \\
\hline & & 30 & $0.0995(0.0019)$ & $0.1120(0.0021)$ & $0.1084(0.0008)$ \\
\hline & & 50 & $0.0995(0.0011)$ & $0.1107(0.0012)$ & $0.1077(0.0005)$ \\
\hline \multirow{3}{*}{$(0.5,2.7563,2)$} & \multirow{3}{*}{0.2} & 10 & $0.1928(0.0118)$ & $0.2235(0.0121)$ & $0.2114(0.0042)$ \\
\hline & & 30 & $0.1973(0.0040)$ & $0.2117(0.0041)$ & $0.2075(0.0018)$ \\
\hline & & 50 & $0.1979(0.0024)$ & $0.2099(0.0024)$ & $0.2052(0.0012)$ \\
\hline \multirow{3}{*}{$(0.5,1.8401,1.5)$} & \multirow{3}{*}{0.3} & 10 & $0.2874(0.0167)$ & $0.3160(0.0165)$ & $0.3103(0.0058)$ \\
\hline & & 30 & $0.2958(0.0056)$ & $0.3095(0.0055)$ & $0.3072(0.0031)$ \\
\hline & & 50 & $0.2977(0.0034)$ & $0.3085(0.0034)$ & $0.3057(0.0020)$ \\
\hline \multirow{3}{*}{$(0.5,1.2943,1.5)$} & \multirow{3}{*}{0.4} & 10 & $0.3854(0.0193)$ & $0.4135(0.0151)$ & $0.4085(0.0067)$ \\
\hline & & 30 & $0.3949(0.0063)$ & $0.4087(0.0060)$ & $0.4051(0.0037)$ \\
\hline & & 50 & $0.3972(0.0037)$ & $0.4059(0.0036)$ & $0.4049(0.0025)$ \\
\hline \multirow{3}{*}{$(1,1.8442,2)$} & \multirow{3}{*}{0.5} & 10 & $0.4819(0.0202)$ & $0.5080(0.0160)$ & $0.5051(0.0072)$ \\
\hline & & 30 & $0.4942(0.0062)$ & $0.5041(0.0062)$ & $0.4990(0.0040)$ \\
\hline & & 50 & $0.4967(0.0037)$ & $0.5022(0.0037)$ & $0.4993(0.0027)$ \\
\hline \multirow{3}{*}{$(1,1.2950,2)$} & \multirow{3}{*}{0.6} & 10 & $0.5856(0.0175)$ & $0.6061(0.0155)$ & $0.5931(0.0038)$ \\
\hline & & 30 & $0.5952(0.0052)$ & $0.5958(0.0051)$ & $0.5994(0.0024)$ \\
\hline & & 50 & $0.5971(0.0031)$ & $0.5987(0.0031)$ & $0.5973(0.0020)$ \\
\hline \multirow{3}{*}{$(1.5,1.3028,2)$} & \multirow{3}{*}{0.7} & 10 & $0.6893(0.0130)$ & $0.6900(0.0121)$ & $0.6902(0.0023)$ \\
\hline & & 30 & $0.6969(0.0037)$ & $0.6921(0.0037)$ & $0.6947(0.0016)$ \\
\hline & & 50 & $0.6980(0.0022)$ & $0.6932(0.0023)$ & $0.6962(0.0012)$ \\
\hline \multirow{3}{*}{$(2.5,1.3123,2)$} & \multirow{3}{*}{0.8} & 10 & $0.7945(0.0075)$ & $0.7951(0.0084)$ & $0.7926(0.0015)$ \\
\hline & & 30 & $0.7986(0.0021)$ & $0.7955(0.0022)$ & $0.7921(0.0011)$ \\
\hline & & 50 & $0.7991(0.0013)$ & $0.7971(0.0013)$ & $0.7959(0.0008)$ \\
\hline \multirow{3}{*}{$(3,0.7214,1.5)$} & \multirow{3}{*}{0.9} & 10 & $0.8999(0.0025)$ & $0.8931(0.0032)$ & $0.8900(0.0012)$ \\
\hline & & 30 & $0.9000(0.0007)$ & $0.8924(0.0007)$ & $0.8930(0.0004)$ \\
\hline & & 50 & $0.9001(0.0004)$ & $0.8980(0.0004)$ & $0.8967(0.0002)$ \\
\hline
\end{tabular}

- In the case where $\theta$ is unknown, the Bayes estimator based on Prior 2 has a smaller MSE than Bayes estimator based on Prior 1 and MLE. Also, there is no difference between the Bayes estimator based on Prior 1 and MLE except for $n=10$. In this sample size, in most cases, the Bayes estimator based on Prior 1 is better than the MLE. However, for extreme values of $R_{s, k}$, the MLE has a smaller MSE.

- As anticipated, with the increase in sample size, the ALs of intervals tend to decrease.

- The ALs of all the intervals are maximized when $R_{s, k}$ is 0.5 and they become smaller and smaller as we go away to extreme values.

- The HPD credible intervals based on Prior 2 have smaller ALs and higher CPs than the HPD credible intervals based on Prior 1 as well as different ACI methods.

- The constructed intervals based on Prior 1 perform better performance than different ACI methods except for extreme values of $R_{s, k}$.

- For extreme values of $R_{s, k}$, it is observed that AST, NT, and LOGT have the lowest to highest ALs respectively, but for moderate values of $R_{s, k}$, it can be seen that LOGT has the best performances among the different ACI methods.

- Comparing the different ACI methods showed that NT, AST, and LOGT have the lowest to highest CPs, respectively. 
- For a large sample size, all different ACI methods show the same ALs.

- In most cases, the CPs of the asymptotic and HPD intervals are respectively lower and upper than the predefined nominal level of $95 \%$.

- In general, it is observed that the performance of the estimators of $R_{s, k}$ is better when the common second shape parameter is known than when its value is unknown.

Table 4. Point estimates of $R_{2,5}$ along with MSEs (presented in parenthesis) when $\theta$ is known $(\theta=5)$.

\begin{tabular}{|c|c|c|c|c|c|c|}
\hline \multirow[b]{2}{*}{$(\alpha, \beta)$} & \multirow[b]{2}{*}{$R_{2,5}$} & \multirow[b]{2}{*}{$n$} & \multicolumn{2}{|c|}{ Classical } & \multicolumn{2}{|c|}{ Bayesian } \\
\hline & & & MLE & UMVUE & Prior 1 & Prior 2 \\
\hline \multirow{3}{*}{$(2,19.3019)$} & \multirow{3}{*}{0.1} & 10 & $0.1141(0.0050)$ & $0.1001(0.0045)$ & $0.1270(0.0054)$ & $0.1078(0.0010)$ \\
\hline & & 30 & $0.1048(0.0014)$ & $0.1001(0.0013)$ & $0.1087(0.0014)$ & $0.1042(0.0005)$ \\
\hline & & 50 & $0.1026(0.0008)$ & $0.1000(0.0008)$ & $0.1059(0.0008)$ & $0.1026(0.0004)$ \\
\hline \multirow{3}{*}{$(2,11.0249)$} & \multirow{3}{*}{0.2} & 10 & $0.2144(0.0101)$ & $0.2005(0.0107)$ & $0.2263(0.0102)$ & $0.2070(0.0024)$ \\
\hline & & 30 & $0.2050(0.0033)$ & $0.1999(0.0034)$ & $0.2090(0.0033)$ & $0.2059(0.0014)$ \\
\hline & & 50 & $0.2033(0.0020)$ & $0.2002(0.0020)$ & $0.2049(0.0020)$ & $0.2036(0.0009)$ \\
\hline \multirow{3}{*}{$(2,7.3606)$} & \multirow{3}{*}{0.3} & 10 & $0.3094(0.0139)$ & $0.3009(0.0155)$ & $0.3173(0.0128)$ & $0.3044(0.0036)$ \\
\hline & & 30 & $0.3034(0.0049)$ & $0.2999(0.0050)$ & $0.3053(0.0047)$ & $0.3031(0.0022)$ \\
\hline & & 50 & $0.3019(0.0029)$ & $0.2996(0.0030)$ & $0.3042(0.0029)$ & $0.3025(0.0016)$ \\
\hline \multirow{3}{*}{$(2,5.1774)$} & \multirow{3}{*}{0.4} & 10 & $0.4038(0.0158)$ & $0.4003(0.0180)$ & $0.4060(0.0144)$ & $0.4034(0.0046)$ \\
\hline & & 30 & $0.4009(0.0056)$ & $0.3997(0.0058)$ & $0.4023(0.0055)$ & $0.4013(0.0029)$ \\
\hline & & 50 & $0.4004(0.0034)$ & $0.4001(0.0035)$ & $0.4012(0.0033)$ & $0.4011(0.0021)$ \\
\hline \multirow{3}{*}{$(3,5.5328)$} & \multirow{3}{*}{0.5} & 10 & $0.4979(0.0161)$ & $0.5004(0.0178)$ & $0.4940(0.0149)$ & $0.4983(0.0050)$ \\
\hline & & 30 & $0.4988(0.0056)$ & $0.5004(0.0058)$ & $0.4974(0.0055)$ & $0.4995(0.0032)$ \\
\hline & & 50 & $0.4990(0.0034)$ & $0.4999(0.0034)$ & $0.4977(0.0034)$ & $0.4995(0.0018)$ \\
\hline \multirow{3}{*}{$(3,3.8853)$} & \multirow{3}{*}{0.6} & 10 & $0.5914(0.0143)$ & $0.6006(0.0155)$ & $0.5852(0.0139)$ & $0.5934(0.0030)$ \\
\hline & & 30 & $0.5970(0.0048)$ & $0.5999(0.0049)$ & $0.5952(0.0047)$ & $0.5975(0.0022)$ \\
\hline & & 50 & $0.5982(0.0029)$ & $0.6001(0.0030)$ & $0.5966(0.0029)$ & $0.5971(0.0019)$ \\
\hline \multirow{3}{*}{$(4,3.4743)$} & \multirow{3}{*}{0.7} & 10 & $0.6878(0.0111)$ & $0.7001(0.0112)$ & $0.6807(0.0109)$ & $0.6926(0.0019)$ \\
\hline & & 30 & $0.6960(0.0035)$ & $0.7000(0.0035)$ & $0.6921(0.0036)$ & $0.6947(0.0017)$ \\
\hline & & 50 & $0.6978(0.0021)$ & $0.7003(0.0021)$ & $0.6960(0.0021)$ & $0.6961(0.0013)$ \\
\hline \multirow{3}{*}{$(5,2.6246)$} & \multirow{3}{*}{0.8} & 10 & $0.7881(0.0065)$ & $0.8000(0.0063)$ & $0.7779(0.0069)$ & $0.7938(0.0013)$ \\
\hline & & 30 & $0.7960(0.0020)$ & $0.8003(0.0019)$ & $0.7920(0.0021)$ & $0.7949(0.0010)$ \\
\hline & & 50 & $0.7979(0.0012)$ & $0.7999(0.0012)$ & $0.7955(0.0012)$ & $0.7968(0.0007)$ \\
\hline \multirow{3}{*}{$(7,1.6834)$} & \multirow{3}{*}{0.9} & 10 & $0.8920(0.0023)$ & $0.9001(0.0018)$ & $0.8840(0.0026)$ & $0.8939(0.0006)$ \\
\hline & & 30 & $0.8972(0.0006)$ & $0.9003(0.0006)$ & $0.8948(0.0007)$ & $0.8968(0.0003)$ \\
\hline & & 50 & $0.8984(0.0004)$ & $0.9000(0.0003)$ & $0.8964(0.0004)$ & $0.8976(0.0002)$ \\
\hline
\end{tabular}

\section{Real data analysis}

In this section, we present two different lifetime data sets to display the application of the proposed approaches in this paper.

\subsection{Real data set $I$}

In this subsection, we study the data set that was initially reported by [36]. The data represent the length of times (in minute) to the breakdown of an insulating fluid at seven voltage levels, ranging from 26 to 38 kilovolts $(\mathrm{kV})$. Here, we consider the time to the breakdown of $36 \mathrm{kV}$ and $38 \mathrm{kV}$. This data set is reported as follows:

$$
\begin{gathered}
\mathrm{X}: 36 \mathrm{kV} \quad 0.35,0.59,0.96,0.99,1.69,1.97,2.07,2.58,2.71,2.90,3.67,3.99, \\
5.35,13.77,25.50
\end{gathered}
$$

$\mathbf{Y}: 38 \mathbf{~ k V} \quad 0.09,0.39,0.47,0.73,0.74,1.13,1.40,2.38$ 


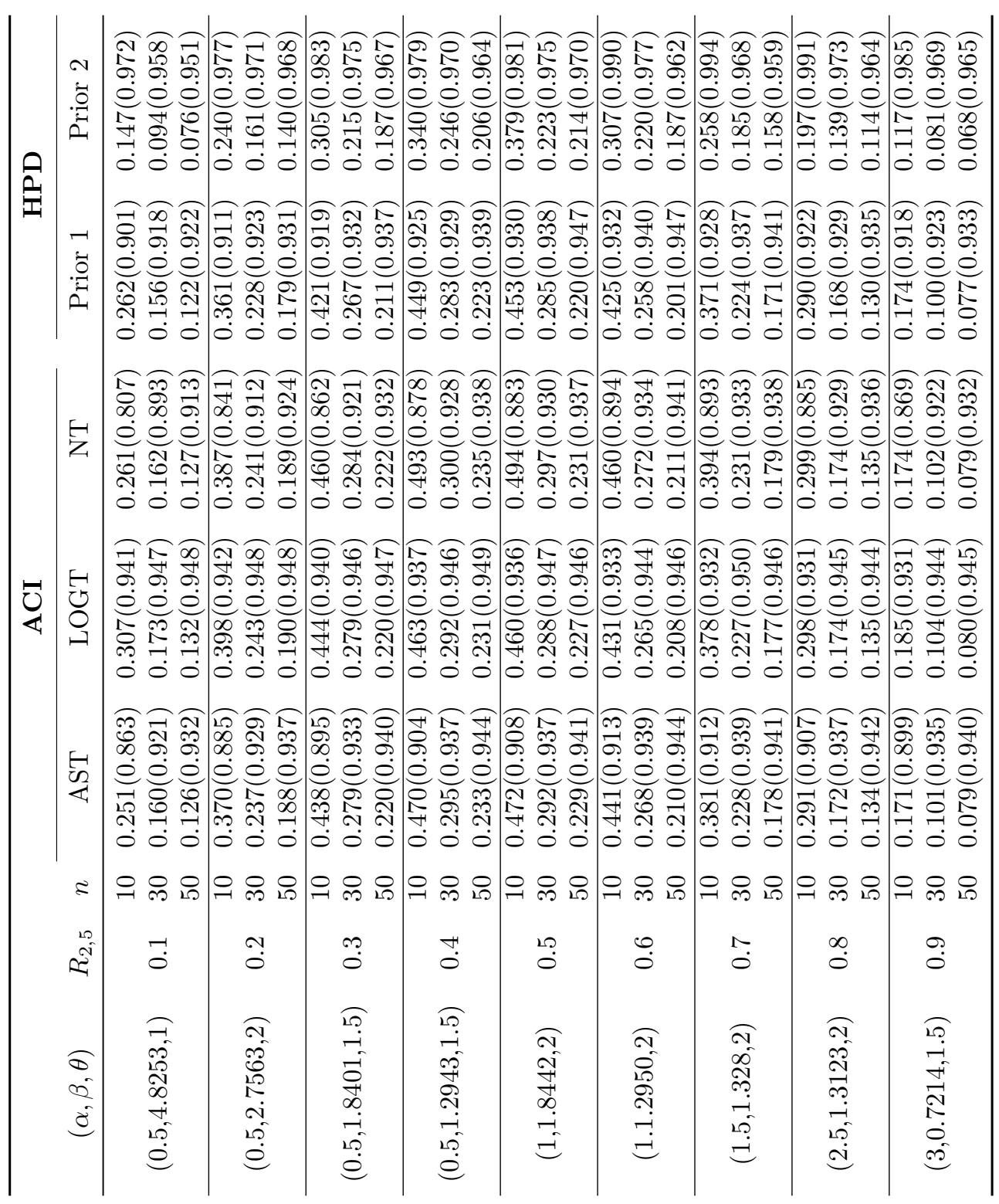




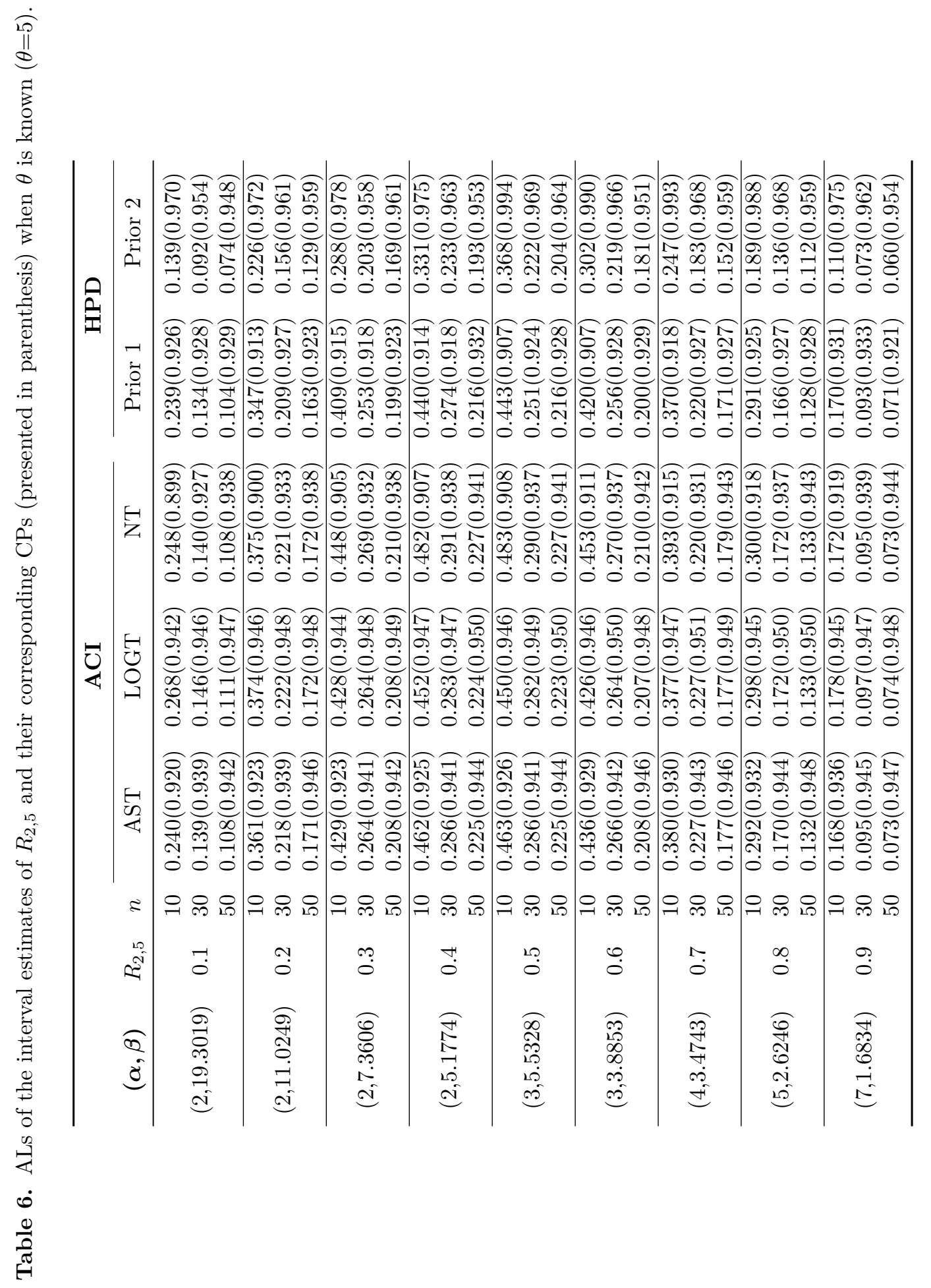


The validity of the IKu distribution for strength data set $\mathbf{X}$ and stress data set $\mathbf{Y}$ is checked by using the Kolmogorov-Smirnov (K-S) test. Furthermore, in this example the $\mathrm{IKu}$ distribution is compared to some well-known lifetime models namely Burr type XII (Burr), modified Lomax (ML)[12], inverse Weibull (IW), inverse Gamma (IG), exponentiated Weibull (EW), and exponentiated Rayleigh (ER) distributions. The probability density function of these distributions are listed below:

$$
\begin{aligned}
& \text { Burr : } f(x ; \alpha, \theta)=\alpha \theta x^{\theta-1}\left(1+x^{\theta}\right)^{-(\alpha+1)}, \\
& \text { ML : } f(x ; \alpha, \theta, \sigma)=\frac{\alpha\left[\theta+1+\log \left(1+\frac{x}{\sigma}\right)\right]\left[\log \left(1+\frac{x}{\sigma}\right)\right]^{\theta}}{\sigma\left(1+\frac{x}{\sigma}\right)\left[1+\log \left(1+\frac{x}{\sigma}\right)\right]^{\theta+1}} \exp \left[-\alpha \frac{\left[\log \left(1+\frac{x}{\sigma}\right)\right]^{\theta+1}}{\left[1+\log \left(1+\frac{x}{\sigma}\right)\right]^{\theta}}\right], \\
& \text { IW }: f(x ; \alpha, \theta)=\theta \alpha^{-\theta} x^{-(\theta+1)} \exp \left[-(\alpha x)^{-\theta}\right] \\
& \text { IG : } f(x ; \alpha, \theta)=\frac{\theta^{\alpha}}{\Gamma(\alpha)}\left(\frac{1}{x}\right)^{\alpha+1} \exp \left[-\frac{\theta}{x}\right] \\
& \text { EW }: f(x ; \alpha, \theta, \sigma)=\frac{\alpha \theta}{\sigma}\left(\frac{x}{\sigma}\right)^{\theta-1} \exp \left(-\left[\frac{x}{\sigma}\right]^{\theta}\right)\left[1-\exp \left(-\left[\frac{x}{\sigma}\right]^{\theta}\right)\right]^{\alpha-1}, \\
& \text { ER : } f(x ; \alpha, \theta)=2 \alpha \theta x \exp \left(-\theta x^{2}\right)\left[1-\exp \left(-\theta x^{2}\right)\right]^{\alpha-1},
\end{aligned}
$$

where $x, \alpha, \theta$ and $\sigma$ are positive except for $\sigma$ parameter in the ML distribution, which is greater than -1 . For purpose of comparison of the above distributions with the IKu distribution, we use several criteria including, Kolmogorov-Smirnov (K-S) distance, Akaike information criterion (AIC) and Bayesian information criterion (BIC). The MLEs of unknown parameters, K-S distances along with P-values, AIC, and BIC for all models and for both $\mathbf{X}$ and $\mathbf{Y}$ data sets are reported in Table 7. According to P-values, all seven models are accepted for fitting this data, but Based on AIC and BIC criteria, it is observed that the IKu distribution provides the better fit compared to the other competitive models. The validity of the IKu distribution is also supported by the various plots shown in Figure 6.

Table 7. The MLEs of unknown parameters along with some measures of goodness-of-fit for real data set I.

\begin{tabular}{cccccccc}
\hline data & Model & $\hat{\boldsymbol{\alpha}}$ & $\hat{\boldsymbol{\theta}}$ & $\hat{\boldsymbol{\sigma}}$ & KS (P-value) & AIC & BIC \\
\hline \multirow{6}{*}{ X } & Burr & 0.3764 & 2.4605 & - & $0.172(0.705)$ & 76.474 & 77.890 \\
& ML & 3.5441 & 9.0926 & 0.1354 & $0.113(0.978)$ & 77.396 & 79.520 \\
& IW & 0.6823 & 1.0280 & - & $0.155(0.813)$ & 76.278 & 77.694 \\
& IG & 1.1221 & 1.6648 & - & $0.159(0.785)$ & 76.174 & 77.590 \\
& EW & 55.196 & 0.2464 & 0.0058 & $0.112(0.981)$ & 77.406 & 79.530 \\
& ER & 0.2878 & 0.0059 & - & $0.301(0.106)$ & 84.330 & 85.746 \\
& IKu & $\mathbf{3 . 7 0 9 8}$ & $\mathbf{1 . 4 9 3 0}$ & - & $\mathbf{0 . 1 1 6}(\mathbf{0 . 9 7 4})$ & $\mathbf{7 5 . 4 7 0}$ & $\mathbf{7 6 . 8 8 6}$ \\
\hline & Burr & 1.6310 & 1.7672 & - & $0.148(0.984)$ & 18.084 & 18.243 \\
& ML & 520.45 & 66.8900 & 0.0005 & $0.156(0.972)$ & 20.052 & 20.290 \\
& IW & 2.5139 & 0.9627 & - & $0.236(0.682)$ & 21.487 & 21.645 \\
Y & IG & 1.1007 & 0.4286 & - & $0.250(0.616)$ & 21.466 & 21.705 \\
& EW & 0.9252 & 1.4263 & 1.0519 & $0.143(0.988)$ & 19.528 & 19.766 \\
& ER & 0.5541 & 0.5093 & - & $0.168(0.951)$ & 17.624 & 17.783 \\
& IKu & $\mathbf{2 . 5 0 1 3}$ & $\mathbf{2 . 7 9 8 2}$ & - & $\mathbf{0 . 1 6 9}(\mathbf{0 . 9 4 7})$ & $\mathbf{1 8 . 5 6 9}$ & $\mathbf{1 8 . 7 2 7}$ \\
\hline
\end{tabular}



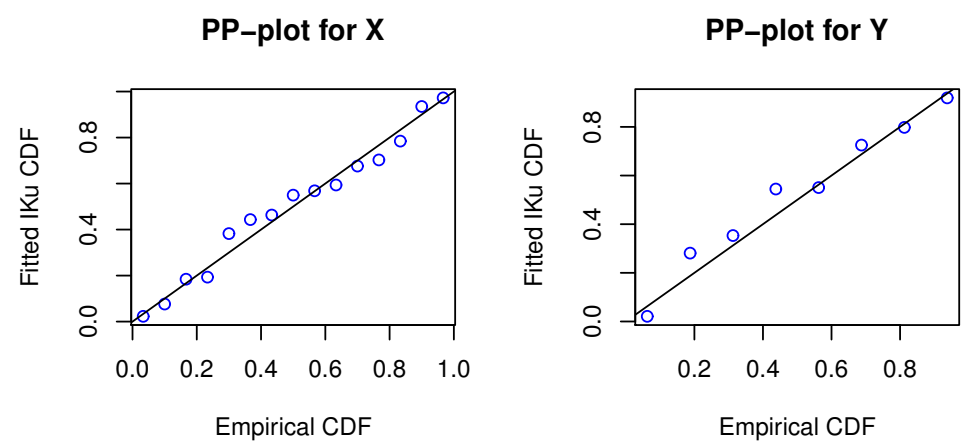

Empirical and fitted PDFs

\section{Empirical and fitted PDFs}
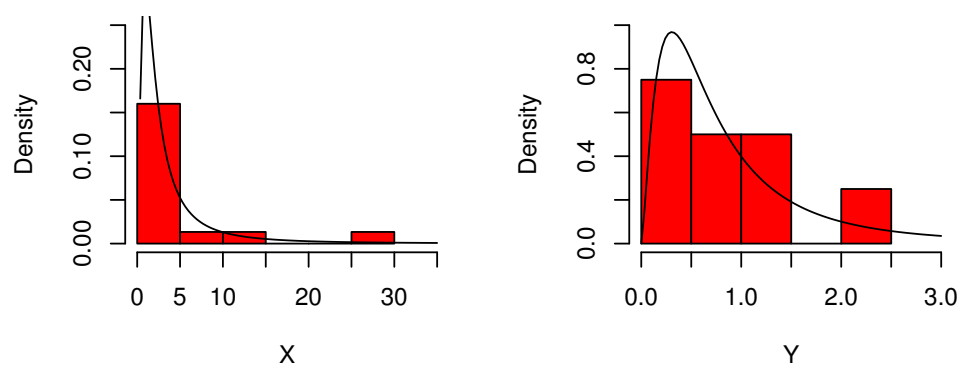

Figure 6. Diagnostic plots for real data set I.

Now, we obtain the reliability of the MSS model through classical and Bayesian methods for $(s, k)=(2,5)$ and $(4,5)$. First, we use the fixed point method to obtain the ML estimates of unknown parameters. The initial value for this technique is determined by drawing the two functions $y=\theta$ and $y=f(\theta)$ and observing their intersection, where $y=f(\theta)$ is defined in Equation (2.9). The result is shown in Figure 7. Based on this figure we see that the approximate initial value for $\theta$ should be between 1.5 and 2 . The ML estimate of $\theta$ is obtained as $\hat{\theta}=1.7142$. So, using Equations (2.7) and (2.8) the ML estimates of $\alpha$ and $\beta$ are computed as $\hat{\alpha}=4.5239$ and $\hat{\beta}=1.5261$, respectively. From the Bayesian view, the priors selection problem for real data set is a challenging topic because there is no prior knowledge of unknown parameters. In this regard, we followed the method of [49]. Based on this method, the MLEs of the unknown parameters are chosen as priors means and variances are very little (say, 1). Also, if there is no available knowledge about the priors, then non-informative prior may be appropriate. In our study, the Bayes estimates are obtained for both informative and non-informative priors. The hyperparameters of $\left(a_{i}, b_{i}\right)=(0.0001,0.0001), i=1,2,3$ are selected for the non-informative prior (Prior 1$)$. Also, the hyperparameters of $\left(a_{1}, b_{1}\right)=(20.4657,4.5239)$, $\left(a_{2}, b_{2}\right)=(2.3290,1.5261)$ and $\left(a_{3}, b_{3}\right)=(2.9385,1.7142)$ are selected for the informative prior (Prior 2) based on the MLEs of unknown parameters. Tables 8 and 9 give the point and interval estimates of $R_{s, k}$, respectively. Based on these results, the point estimates of $R_{s, k}$ which are obtained based on ML and Bayes approaches are about the same. In view of interval estimates, the constructed intervals based on Bayes estimators have better average lengths than different ACI methods. Also, comparing the different ACI methods observed that AST and LOGT have respectively the lowest and highest average lengths, when $(s, k)=(2,5)$ and LOGT and NT have respectively the lowest and highest average lengths, when $(s, k)=(4,5)$. These results confirm the findings of the simulation. 


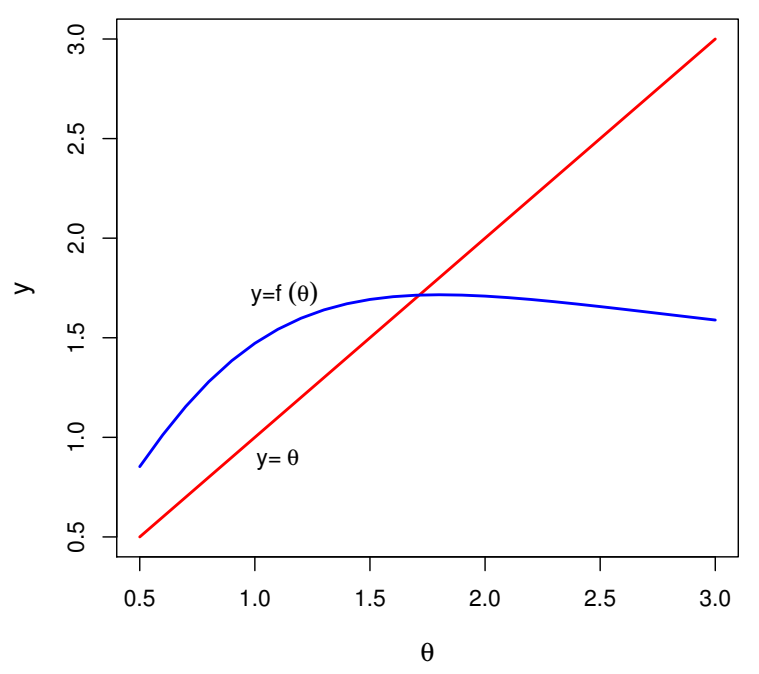

Figure 7. The initial value in the fixed point technique for real data set I.

Table 8. Point estimates of $R_{s, k}$ for real data set I.

\begin{tabular}{cccc}
\hline & & \multicolumn{2}{c}{ Bayes estimates } \\
\cline { 3 - 4 }$(s, k)$ & MLE & Prior 1 & Prior 2 \\
\hline$(2,5)$ & 0.8639 & 0.8583 & 0.8621 \\
$(4,5)$ & 0.6645 & 0.6603 & 0.6657 \\
\hline
\end{tabular}

Table 9. Interval estimates of $R_{s, k}$ for real data set I.

\begin{tabular}{ccccccc}
\hline & \multicolumn{3}{c}{ ACI } & & \multicolumn{2}{c}{ HPD } \\
\cline { 2 - 3 } \cline { 6 - 7 }$(s, k)$ & AST & LOGT & NT & & Prior 1 & Prior 2 \\
\hline$(2,5)$ & $(0.739,0.953)$ & $(0.716,0.941)$ & $(0.756,0.972)$ & & $(0.746,0.956)$ & $(0.770,0.942)$ \\
AL & 0.214 & 0.225 & 0.216 & & 0.210 & 0.172 \\
$(4,5)$ & $(0.426,0.865)$ & $(0.415,0.847)$ & $(0.436,0.893)$ & & $(0.437,0.865)$ & $(0.484,0.840)$ \\
AL & 0.439 & 0.432 & 0.457 & & 0.428 & 0.356 \\
\hline
\end{tabular}

In the first real data set, the underlying data structure for random stress and strength samples is considered as a vector in the MSS model. Such a structure for data is observed in many studies. For more details, one can refer to [3,23,37-40]. On the other hand, there are other studies that the basic data structure for random stress and strength samples in the above model is considered as a matrix. In this case, the observed samples for strength $(\mathbf{X})$ and stress $(\mathbf{Y})$ variables are as follows:

$$
\mathbf{X}=\left[\begin{array}{cccc}
X_{1,1} & X_{1,2} & \cdots & X_{1, k} \\
X_{2,1} & X_{2,2} & \cdots & X_{2, k} \\
\vdots & \vdots & \ddots & \vdots \\
X_{n, 1} & X_{n, 2} & \cdots & X_{n, k}
\end{array}\right], \quad \mathbf{Y}=\left[\begin{array}{c}
Y_{1} \\
Y_{2} \\
\vdots \\
Y_{n}
\end{array}\right]
$$


Although the above structure for strength and stress data is more consistent with the MSS model structure, the estimate of the $R_{s, k}$ will ultimately be exactly the same under both data structures. The matrix $\mathbf{X}$ with $n$ rows and $k$ columns convert to a vector with $n k$ element. Also, the matrix $\mathbf{Y}$ with $n$ rows and 1 column convert to a vector with $n$ element. This process is illustrated in the following real data set II.

\subsection{Real data set II}

In this subsection, we consider the monthly rainfall of long beach airport in California, USA, from 2005 to 2017, to construct a scenario concerning the excessive drought. The complete data are also available at http://www.laalmanac.com/weather/we10aa.php. According to the Meteorological Organization, January, February, March, and December are the months with the highest rainfall. We claim that if the rainfall of the long beach airport, for at least two months from the mentioned months, is more than the annual average rainfall of the previous year, there will be no excessive drought in the month of summer afterward. This can be described as a 2-out-of-4 G system. It should be stated that [31] have studied these data. Let $Y_{1}$ denotes the mean annual rainfall for the 2005-2006 season and $X_{1 k}, k=1,2, \ldots, 4$, are the monthly rainfall from December 2006 to March 2007. Similarly, let $Y_{2}$ be the mean annual rainfall for the 2007-2008 season and $X_{1 k}, k=1,2, \ldots, 4$, are the monthly rainfall from December 2008 to March 2009. Here, a season represents the period from the beginning of July to the end of June. When we carry on this data process up to $2016-2017$, we get $n=6$. The real data set for strength and stress variables are as follows:

$$
\mathbf{X}=\left[\begin{array}{llll}
0.68 & 0.20 & 0.49 & 0.03 \\
2.61 & 0.17 & 4.04 & 0.42 \\
2.61 & 0.17 & 4.04 & 0.42 \\
2.40 & 1.04 & 0.30 & 0.85 \\
4.41 & 0.87 & 0.24 & 0.49 \\
3.59 & 9.33 & 4.72 & 0.15
\end{array}\right], \quad \mathbf{Y}=\left[\begin{array}{l}
0.718 \\
0.951 \\
1.305 \\
0.631 \\
0.375 \\
0.540
\end{array}\right]
$$

For computational purposes, we treat each of the $\mathbf{X}$ and $\mathbf{Y}$ matrices as vectors. Thus, we obtain the vector $\mathbf{X}$ with 24 elements $(m=24)$ and the vector $\mathbf{Y}$ with 6 elements $(n=6)$. We check whether strength data $\mathbf{X}$ and stress data $\mathbf{Y}$ come from IKu distribution or not by the goodness-of-fit test as the same approach in the previous data. Table 10 presents the MLEs of unknown parameters, K-S distances along with P-values, AIC, and BIC for each model and data set. Based on this table, it can be seen that the IKu distribution worked better than the other distributions with the minimum AIC and BIC. The validity of the IKu distribution is also supported by the various plots shown in Figure 8.

In a similar way to the previous data set, we obtain the reliability of the MSS model for $(s, k)=(2,4)$. The ML estimates of $\alpha, \beta$, and $\theta$ are obtained as 1.5560, 1.7215 and 1.6045 , respectively. Then, the MLE of $R_{2,4}$ is obtained as 0.5723 . From the Bayesian view, the hyperparameters of $\left(a_{i}, b_{i}\right)=(0.0001,0.0001), i=1,2,3$ are selected for the non-informative prior (Prior 1). Also, the hyperparameters of $\left(a_{1}, b_{1}\right)=(2.4211,1.5560)$, $\left(a_{2}, b_{2}\right)=(2.9636,1.7215)$ and $\left(a_{3}, b_{3}\right)=(2.5744,1.6045)$ are selected for the informative prior (Prior 2) using the method of [49]. The Bayes estimates of $R_{2,4}$ based on Prior 1 and Prior 2 are computed as 0.5814 and 0.5770 , respectively. Table 11 gives the interval estimates of $R_{2,4}$. In view of interval estimates, the constructed intervals based on the Bayes estimator under Prior 2 has a better average length than the Bayes estimator under Prior 1 as well as better than different ACI methods. Also, comparing the different ACI methods observed that LOGT and NT have the lowest and highest average lengths, respectively. 
Table 10. The MLEs of unknown parameters along with some measures of goodness-of-fit for real data set II.

\begin{tabular}{cccccccc}
\hline data & Model & $\hat{\boldsymbol{\alpha}}$ & $\hat{\boldsymbol{\theta}}$ & $\hat{\boldsymbol{\sigma}}$ & KS (P-value) & AIC & BIC \\
\hline \multirow{6}{*}{} & Burr & 1.0342 & 1.1883 & - & $0.126(0.799)$ & 88.766 & 91.122 \\
& ML & 36.990 & -0.1866 & 161.61 & $0.100(0.949)$ & 89.760 & 93.294 \\
& IW & 2.1355 & 0.6731 & - & $0.117(0.863)$ & 93.358 & 95.714 \\
& IG & 0.5704 & 0.1864 & - & $0.146(0.634)$ & 97.726 & 99.082 \\
& EW & 2.8759 & 0.4753 & 0.4396 & $0.099(0.956)$ & 89.182 & 92.716 \\
& ER & 0.2678 & 0.0293 & - & $0.167(0.467)$ & 90.688 & 93.044 \\
& IKu & $\mathbf{1 . 3 4 9 5}$ & $\mathbf{1 . 3 4 2 4}$ & - & $\mathbf{0 . 1 2 3}(\mathbf{0 . 8 1 7})$ & $\mathbf{8 8 . 5 3 0}$ & $\mathbf{9 0 . 8 8 6}$ \\
\hline \multirow{6}{*}{} & Burr & 2.4130 & 3.3177 & - & $0.166(0.985)$ & 6.080 & 5.664 \\
& ML & 0.0038 & 2.9248 & 0.1509 & $0.180(0.970)$ & 7.980 & 7.355 \\
Y & IW & 1.7496 & 2.7483 & - & $0.144(0.998)$ & 6.028 & 5.612 \\
& IG & 6.6044 & 4.2543 & - & $0.123(0.999)$ & 5.658 & 5.242 \\
& EW & 27.837 & 0.7747 & 0.1267 & $0.123(0.999)$ & 7.622 & 6.997 \\
& ER & 1.9372 & 2.2513 & - & $0.184(0.963)$ & 5.960 & 5.544 \\
& IKu & $\mathbf{2 6 . 7 8 4}$ & $\mathbf{7 . 0 8 3 4}$ & - & $\mathbf{0 . 1 2 3 ( 0 . 9 9 9 )}$ & $\mathbf{5 . 6 9 4}$ & $\mathbf{5 . 2 7 8}$ \\
\hline
\end{tabular}

\section{P-P plot for $X$}

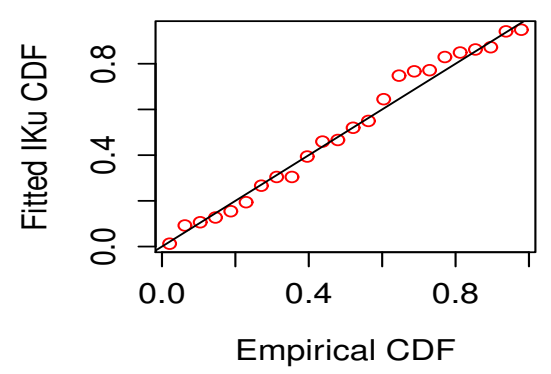

Empirical and fitted PDFs

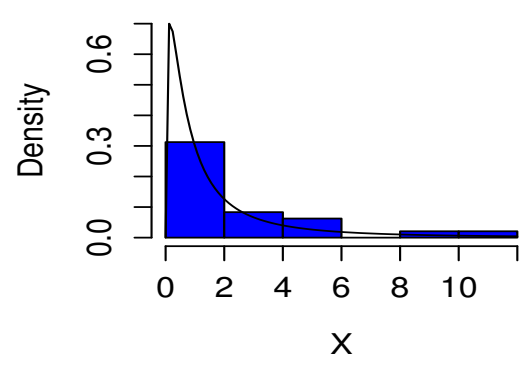

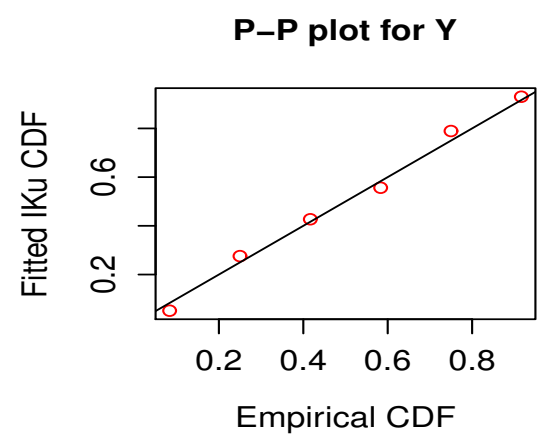

Empirical and fitted PDFs

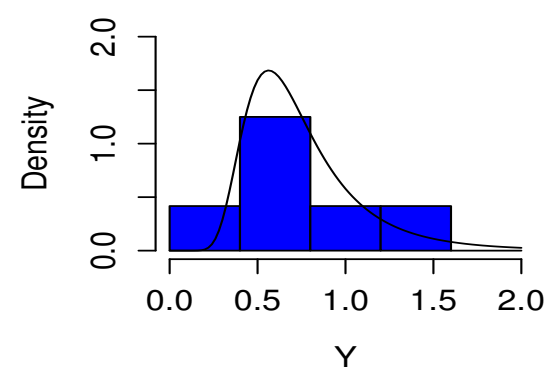

Figure 8. Diagnostic plots for real data set II.

Table 11. Interval estimates of $R_{s, k}$ for real data set II.

\begin{tabular}{ccccccc}
\hline & \multicolumn{3}{c}{ ACI } & & \multicolumn{2}{c}{ HPD } \\
\cline { 2 - 4 } \cline { 6 - 7 }$(s, k)$ & AST & LOGT & NT & & Prior 1 & Prior 2 \\
\hline$(2,4)$ & $(0.323,0.803)$ & $(0.324,0.788)$ & $(0.322,0.823)$ & & $(0.349,0.816)$ & $(0.369,0.774)$ \\
AL & 0.480 & 0.464 & 0.501 & & 0.467 & 0.405 \\
\hline
\end{tabular}




\section{Concluding remarks}

In this article, we have considered the reliability of the MSS model under the assumption that the stress and strength random variables are taken from IKu distributions. The reliability of the MSS model is obtained using the MLE and approximation Bayes estimate when the common second shape parameter is unknown. Also, it is obtained explicitly using the MLE, UMVUE, and Bayes estimate, when the common second shape parameter is known. The asymptotic and HPD intervals are constructed. Furthermore, two other asymptotic confidence intervals are derived based on Logit and Arcsin transformations.

The simulation results showed that the bias and MSE of $R_{s, k}$ decrease as the sample size increases. Also, the ALs of interval estimates get shorter when the sample size increases. According to the MSE, AL, and CP values, the Bayesian estimators based on informative priors had the best performances among the estimators. Moreover, the MSEs and ALs of all estimators were low when $R_{s, k}$ tends to the extreme value and they were high when $R_{s, k}$ tends to moderate value. Comparing the different ACI approaches in terms of the ALs indicated that the Arcsin transformation and Logit transformation worked better respectively for the extreme and moderate values of $R_{s, k}$.

\section{References}

[1] A.M. Abd AL-Fattah, A.A. El-Helbawy and G.R. Al-Dayian, Inverted Kumaraswamy distribution: Properties and estimation, Pakistan. J. Stat. Oper. Res. 33 (1), 37-61, 2017.

[2] M.H. Abu-Moussa and M.M.M. El-Din, On estimation and prediction for the inverted Kumaraswamy distribution based on general progressive censored samples, Pakistan. J. Stat. Oper. Res. 14 (3), 717-736, 2018.

[3] F.G. Akgül, Reliability estimation in multicomponent stressstrength model for ToppLeone distribution, J. Stat. Comput. Simul. 89 (15), 2914-2929, 2019.

[4] F.G. Akgül, Classical and Bayesian estimation of multicomponent stressstrength reliability for exponentiated Pareto distribution, Soft Comput. 25 (14), 9185-9197, 2021.

[5] F.G. Akgül and B. Şenoğlu, Estimation of $P(X<Y)$ using ranked set sampling for the Weibull distribution, Qual. Technol. Quant. Manag. 14 (3), 296-309, 2017.

[6] D.K. Al-Mutairi, M.E. Ghitany and D. Kundu, Inferences on stress-strength reliability from Lindley distributions, Comm. Statist. Theory Methods 42 (8), 1443-1463, 2013.

[7] B. Al-Zahrani and S. Basloom, Estimation of the stress-strength reliability for the Dagum distribution, J. Adv. Stat 1 (3), 157-170, 2016.

[8] X. Bai, Y. Shi, Y. Liu and B. Liu, Reliability inference of stressstrength model for the truncated proportional hazard rate distribution under progressively Type-II censored samples, Appl. Math. Model. 65, 377-389, 2019.

[9] M. Basirat, S. Baratpour and J. Ahmadi, On estimation of stressstrength parameter using record values from proportional hazard rate models, Comm. Statist. Theory Methods 45 (19), 5787-5801, 2016.

[10] G.K. Bhattacharyya and R.A. Johnson, Estimation of reliability in a multicomponent stress-strength model, J. Amer. Statist. Assoc. 69 (348), 966-970, 1974.

[11] Z.W. Birnbaum, On a use of the Mann-Whitney statistic, in: Proceedings of the 3rd Berkeley Symp. Math. Statist. Prob, 1, 13-17, 1956.

[12] S. Chattopadhyay, T. Chakraborty, K. Ghosh and A.K. Das, Modified Lomax model: A heavy-tailed distribution for fitting large-scale real-world complex networks, Soc. Netw. Anal. Min 11 (1), 1-24, 2021.

[13] M.H. Chen and Q.M. Shao, Monte Carlo estimation of Bayesian credible and HPD intervals, J. Comput. Graph. Statist. 8 (1), 69-92, 1999. 
[14] S. Dey, J. Mazucheli and M.Z. Anis, Estimation of reliability of multicomponent stressstrength for a Kumaraswamy distribution, Comm. Statist. Theory Methods 46 (4), 1560-1572, 2017.

[15] B. Efron, Logistic regression, survival analysis, and the Kaplan-Meier curve, J. Amer. Statist. Assoc. 83 (402), 414-425, 1988.

[16] S. Foss, D. Korshunov and S. Zachary, Heavy-tailed and long-tailed distributions, An Introduction to Heavy-Tailed and Subexponential Distributions, 7-42, Springer, 2013.

[17] A. Gelman, J.B. Carlin, H.S. Stern and D.B. Rubin, Bayesian Data Analysis, Chapman and Hall, 2003.

[18] A.I. Genc, Estimation of $P(X>Y)$ with ToppLeone distribution, J. Stat. Comput. Simul. 83 (2), 326-339, 2013.

[19] M.E. Ghitany, D.K. Al-Mutairi and S.M. Aboukhamseen, Estimation of the reliability of a stress-strength system from power Lindley distributions, J. Stat. Comput. Simul. 44 (1), 118-136, 2015.

[20] F. Jamal, M. Arslan Nasir, G. Ozel, M. Elgarhy and N. Mamode Khan, Generalized inverted Kumaraswamy generated family of distributions: Theory and applications, J. Appl. Stat. 46 (16), 2927-2944, 2019.

[21] M.K. Jha, S. Dey and Y.M. Tripathi, Reliability estimation in a multicomponent stressstrength based on unit-Gompertz distribution, Int. J. Qual. Reliab. Manag 37 (3), 428-450, 2019.

[22] M.K. Jha, Y.M. Tripathi and S. Dey, Multicomponent stress-strength reliability estimation based on unit generalized Rayleigh distribution, Int. J. Qual. Reliab. Manag 38 (10), 2048-2079, 2021.

[23] M. Jovanovic, B. Milosevic and M. Obradovic, Estimation of stress-strength probability in a multicomponent model based on geometric distribution, Hacet. J. Math. Stat. 49 (4), 1515-1532, 2020.

[24] T. Kayal, Y.M. Tripathi, S. Dey and S.J. Wu, On estimating the reliability in a multicomponent stress-strength model based on Chen distribution, Comm. Statist. Theory Methods 49 (10), 2429-2447, 2020.

[25] F. Kizılaslan, Classical and Bayesian estimation of reliability in a multicomponent stress-strength model based on the proportional reversed hazard rate mode, Math. Comput. Simulation 38 (2), 36-62, 2017.

[26] F. Kizılaslan and M. Nadar, Estimation of reliability in a multicomponent stressstrength model based on a bivariate Kumaraswamy distribution, Statist. Papers 59 (1), 307-340, 2018.

[27] A. Kohansal and S. Shoaee, Bayesian and classical estimation of reliability in a multicomponent stress-strength model under adaptive hybrid progressive censored data, Statist. Papers 62 (1), 309-359, 2021.

[28] P. Kumaraswamy, A generalized probability density function for double-bounded random processes, J. Hydrol. 46 (1-2), 79-88, 1980.

[29] A.O. Langlands, S.J. Pocock, G.R. Kerr and S.M. Gore, Long-term survival of patients with breast cancer: a study of the curability of the disease, Br. Med. J. 2 (6200), 12471251, 1979.

[30] F. Louzada, P.L. Ramos and D. Nascimento, The inverse Nakagami-m distribution: a novel approach in reliability, IEEE Trans. Rel. 67 (3), 1030-1042, 2018.

[31] A.K. Mahto and Y.M. Tripathi, Estimation of reliability in a multicomponent stressstrength model for inverted exponentiated Rayleigh distribution under progressive censoring, OPSEARCH 57, 1043-1069, 2020.

[32] A.K. Mahto, Y.M. Tripathi and F. Kuzllaslan, Estimation of reliability in a multicomponent stressstrength model for a general class of inverted exponentiated distributions under progressive censoring, J. Stat. Theory Pract. 14 (4), 1-35, 2020. 
[33] R.K. Maurya and Y.M. Tripathi, Reliability estimation in a multicomponent stressstrength model for Burr XII distribution under progressive censoring, Braz. J. Probab. Stat. 34 (2), 345-369, 2020.

[34] H.Z. Muhammed, On a bivariate generalized inverted Kumaraswamy distribution, Phys. A: Stat. Mech. Appl. 553, 1-14, 2020.

[35] S.P. Mukherjee and S.S. MAITI, Stress-strength reliability in the Weibull case, Frontiers in Reliability, World Scientific, Singapore, 4, 231-248, 1998.

[36] W.B. Nelson, Applied Life Data Analysis, John Wiley and Sons, 2003.

[37] A. Pak, N.B. Khoolenjani and M.K. Rastogi, Bayesian inference on reliability in a multicomponent stress-strength bathtub-shaped model based on record values, Pakistan. J. Stat. Oper. Res. 15 (2), 431-444, 2019.

[38] A. Pak, A. Kumar Gupta and N. Bagheri Khoolenjani, On reliability in a multicomponent stress-strength model with power Lindley distribution, Rev. Colombiana Estadist. 41 (2), 251-267, 2018.

[39] G.S. Rao, M. Aslam and D. Kundu, Burr-XII distribution parametric estimation and estimation of reliability of multicomponent stress-strength, Comm. Statist. Theory Methods 44 (23), 4953-4961, 2015.

[40] M. Rasekhi, M.M. Saber and H.M. Yousof, Bayesian and classical inference of reliability in multicomponent stress-strength under the generalized logistic model, Comm. Statist. Theory Methods 50 (21), 51145125, 2020.

[41] S. Rezaei, R.A. Noughabi and S. Nadarajah, Estimation of stress-strength reliability for the generalized Pareto distribution based on progressively censored samples, Ann. Data Sci. 2 (1), 83-101, 2015.

[42] J. Rojo, On the preservation of some pure-tail orderings by reliability operations, Stat. Probab. Lett. 17 (3), 189-198, 1993.

[43] J. Rojo, On tail categorization of probability laws, J. Amer. Statist. Assoc. 91 (433), 378-384, 1996.

[44] E.F. Schuster, Classification of probability laws by tail behavior, J. Amer. Statist. Assoc. 79 (388), 936-939, 1984.

[45] V.K. Sharma, Bayesian analysis of head and neck cancer data using generalized inverse Lindley stressstrength reliability model, Comm. Statist. Theory Methods 47 (5), 1155-1180, 2018.

[46] V.K. Sharma, S.K. Singh, U. Singh and F. Merovci, The generalized inverse Lindley distribution: a new inverse statistical model for the study of upside-down bathtub data, Comm. Statist. Theory Methods 45 (19), 5709-5729, 2016.

[47] S.K. Singh, U. Singh and M. Kumar, Bayesian estimation for Poisson-exponential model under progressive type-II censoring data with binomial removal and its application to ovarian cancer data, Comm. Statist. Simulation Comput. 45 (9), 3457-3475, 2016.

[48] H. Xu, S. Foss and Y. Wang, Convolution and convolution-root properties of longtailed distributions, Extremes 18 (4), 605-628, 2015.

[49] A.S. Yadav, M. Saha, S.K. Singh and U. Singh, Bayesian estimation of the parameter and the reliability characteristics of xgamma distribution using Type-II hybrid censored data, Life. Cycle. Reliab. Saf. Eng. 8 (1), 1-10, 2019.

\section{Appendix A.}

The below program is performed in R to obtain the MLE of multicomponent stressstrength reliability based on the fixed point method. The theta is the initial value for the parameter.

This code generates a random sample of size $n$ from $I K u(\alpha, \theta)$. 


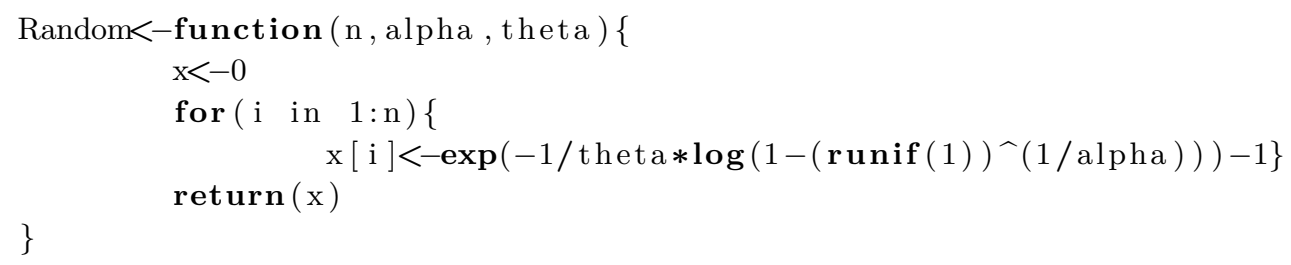

This code calculates the $R_{s, k}$ based on Equation (2.1).

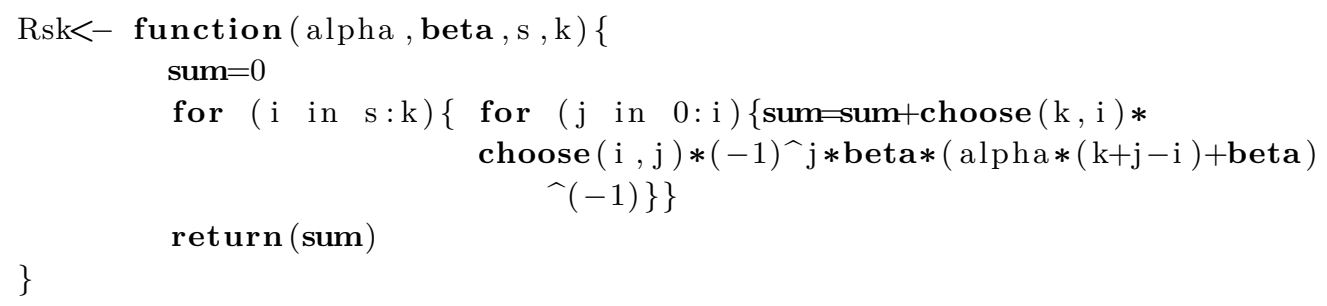

This code determines the MLEs of unknown parameters according to Equations (2.7), (2.8), and (2.10).

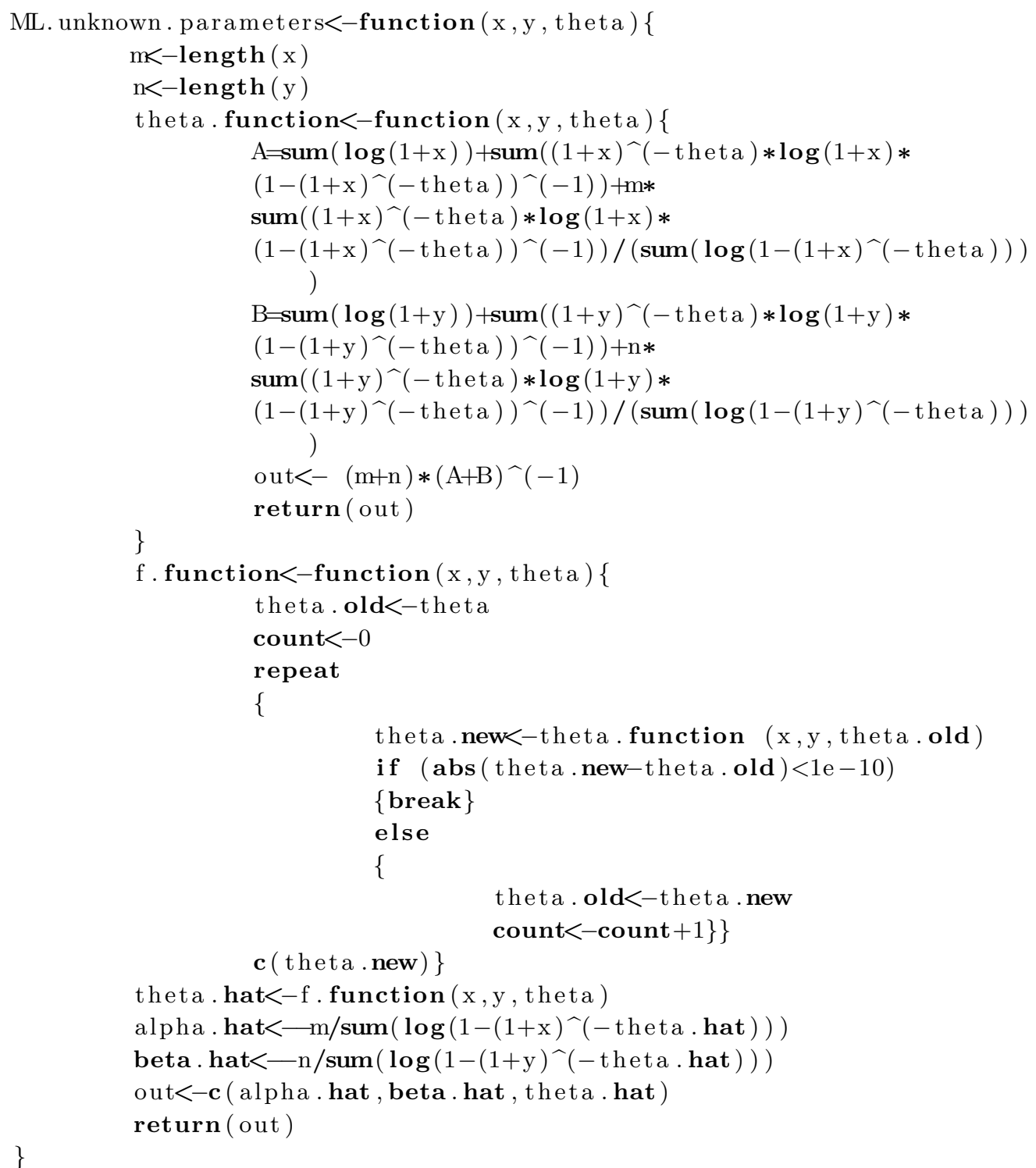

This code estimates the MLE of $R_{s, k}$ with 10000 replication for different combinations of sample sizes of $(m, n)=(10,10),(30,30)$, and $(50,50)$. 


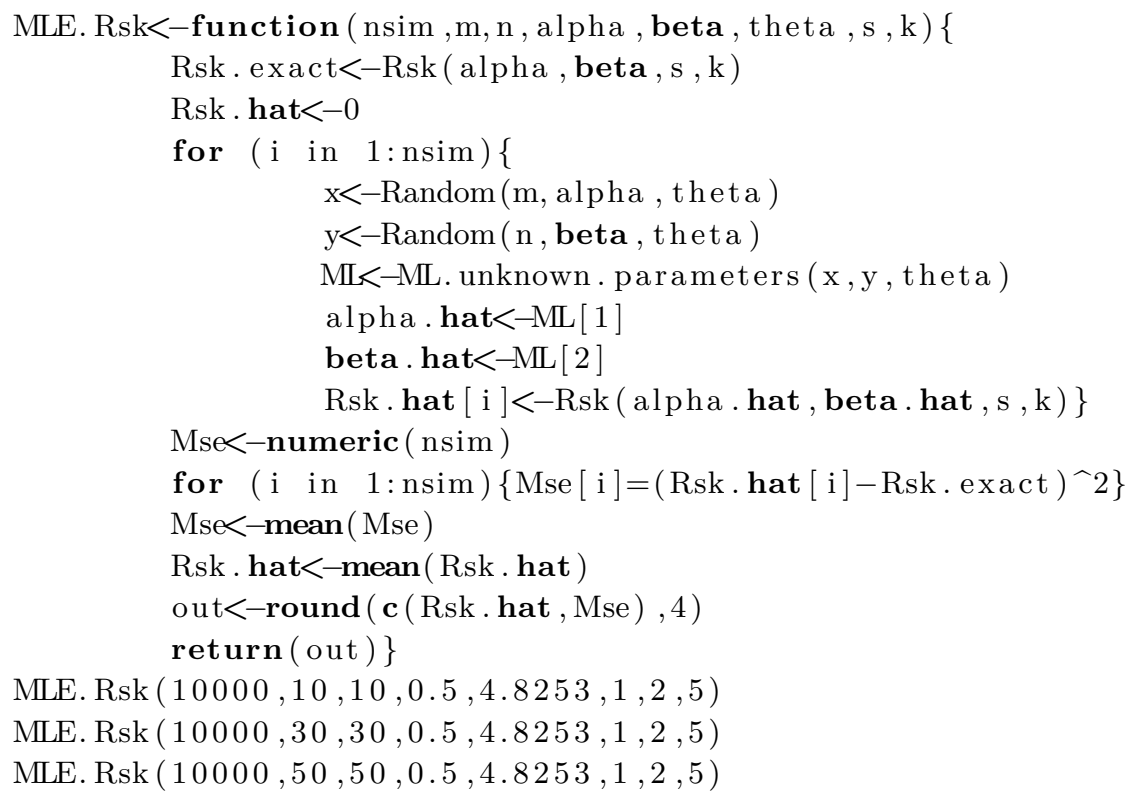

\section{Appendix B.}

The below program determines the MLEs of unknown parameters using the nlminb function in $\mathrm{R}$ software. The alpha, beta, and theta are initial values for the parameters.

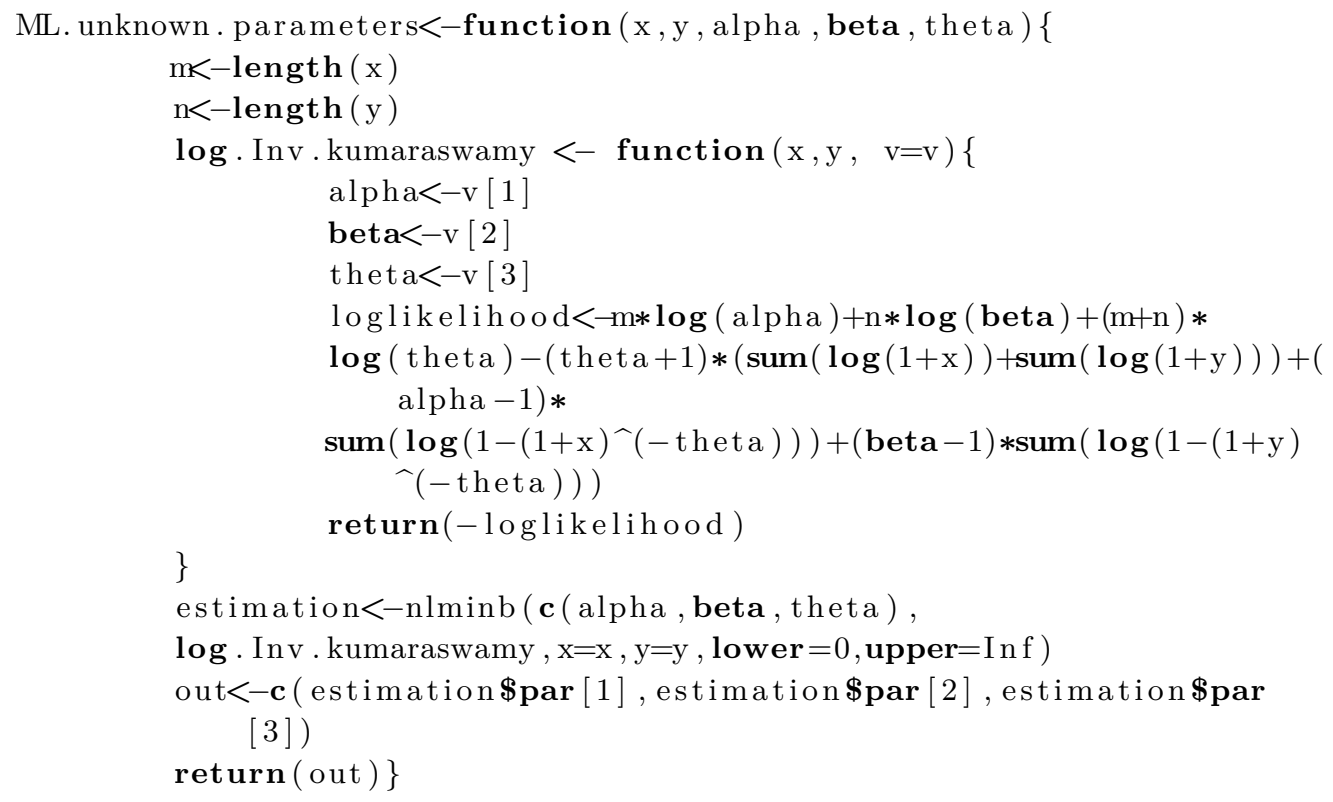

